\title{
Effects of Phase Configuration on Nonlinear Dynamic Characteristics of the Multi-Stage Gear Transmission System
}

Wei Li ( $\square$ liwei@me.ustb.edu.cn )

University of Science and Technology Beijing https://orcid.org/0000-0001-5117-9221

\section{Ziyuan Li}

University of Science and Technology Beijing

Jiapeng Yu

University of Science and Technology Beijing

\section{Research Article}

Keywords: Multi-stage gear transmission, Phase configuration, Nonlinear dynamic, Optimal phase analysis

Posted Date: January 20th, 2022

DOI: https://doi.org/10.21203/rs.3.rs-1137610/v1

License: (a) (1) This work is licensed under a Creative Commons Attribution 4.0 International License. Read Full License 


\title{
Effects of phase configuration on nonlinear dynamic characteristics of the multi-stage gear transmission system
}

Wei $\mathrm{Li}^{*}$, Ziyuan $\mathrm{Li}$, Jiapeng Yu

School of Mechanical Engineering, University of Science and Technology Beijing, 30 Xueyuan Road, Haidian District, 100083 Beijing, China

E-mail: liwei@me.ustb.edu.cn

ORCID: 0000-0001-5117-9221

\begin{abstract}
In the vibration detection of multi-stage gear transmission, the gear ratio has a regular influence on its vibration characteristics. To explore this phenomenon, this paper presents the phase configuration method of the multi-stage gear transmission. The complete mesh phasing space based on the system gear ratio and the phase between each stage is derived. Taking The two-parallel shaft gear transmission system as an example, A nonlinear dynamic model is established. The influence of phase configuration on the vibration characteristics is analyzed in detail. By means of the numerical calculation method, the vibration equation is solved for the gear system, and the varying regularity of the optimal phase angle of the vibration characteristics at different mesh frequency was found. Finally, it reveals the influence of different gear ratios on the vibration characteristics of multi-stage gear transmission.
\end{abstract}

Keyword: Multi-stage gear transmission; Phase configuration; Nonlinear dynamic; Optimal phase analysis

\section{Introduction}

Over the last few years, multi-stage gear transmission has obtained widespread attention and used in various applications such as machine tools, the automotive industry, or wind turbines. In the actual vibration detection of a multi-stage gear reducer, the gear ratio has a regularity influence on system vibration. This paper studies the influence of phase and gear ratio on the dynamics of multi-stage gear transmission.

In the study of multi-stage gear transmission dynamics, the influence of errors and the nonlinear dynamic behavior are of crucial importance[1]. Recently, the influence of temperature on vibration characteristics has been considered[2-5]. there have been many studies on the vibration characteristics of multi-stage gear transmissions. Raclot [6] introduced a mathematical model for the two-stage gear transmission system to simulate the influence of shape deviation and installation error on the dynamic characteristics of the gear system and extended its method to the dynamic coupling of the two-stage gear. Brecher [7] analyzed the dynamic noise characteristics of the two-stage cylindrical gearbox and pointed out that the stiffness of the intermediate shaft and the number of teeth of the two-stage gear has a greater influence on its noise, and the number of teeth of the two-stage gear is the main parameter that affects the phase. Wang [8] established a dimensionless dynamic model for the two-stage fixed shaft and one-stage planetary gear system, and studied the influence of sub-harmonic resonance and crack failure on the stability of the system. Stable motion becomes chaotic motion. Yi [9] considered the effect of induction motors on multi-stage gear systems and concluded that the magnetic field of the motor changes the low frequency torsional characteristics of the system. Li [12] studied the effect of some parameters on the dynamic characteristics of the system for a two-stage parallel shaft gearing system, but did not reflect the non-linear characteristics of the system. Yavuz [10] verified the applicability of the harmonic balance method for the nonlinear dynamics analysis of complex multistage gear systems. Lu [11] modelled the dynamics of a real multi-stage gear system, focusing on the frequency components of the various parts of the model and comparing it with experiments, where 
the model was in good agreement with the experiments. Zhang [12] gave the error transmission model of the multi-stage gear transmission system based on the assembly error of the system. Numerical results are validated against experiment to provide a basis for error-based dynamics analysis of error.

The meshing stiffness has a strong influence on the dynamic characteristics of gears[13, 14] , and there has been some research into the phase of the meshing stiffness. The purpose of dynamic research is to reduce vibration and noise, and the emerging particle damping technology has a good effect[15, 16]. Guo [17] studied the mesh phasing relationship of general compound planetary gears and deduced the relative phase between any two gear meshes in the compound planetary gear when multiple meshing cycles are allowed. When Wang [18] further studied the vibration characteristics of spur planetary gears, it was found that the influence of the mesh phasing on wave vibration is mainly reflected in the number of teeth, the number of planets, their greatest common factor, and the position of the planet's circumference. Peng [19, 20] proposed a method for diagnosing planetary gears based on the mesh phasing, which is related to the theory of the derivation of the mesh phasing of the planetary gear system. Wang [21] developed an analytical dynamic model for high-speed and light planetary gears and performed a spectrum analysis based on the mesh phasing. Sanchez-Espiga [22] proposed a transmission load sharing method based on the mesh phasing in planetary gear transmission, which shows that phase is widely used in gear transmission systems.

In summary, the dynamics research of the multi-stage gear transmission system is mainly in various errors and nonlinear dynamic behaviors. The multi-stage structure is represented by a twostage fixed shaft, planetary and bevel gear transmission. Although a few researchers have studied the load sharing of planetary gear transmission, the research on the phase and gear ratio of multi-stage gear transmission is scarcely few.

This paper presents a method of the meshing space of the multi-stage gear transmission system based on the phase configuration. A nonlinear dynamic model of the two-stage gear transmission system based on phase configuration is established. The nonlinear dynamic equations of phase configuration are derived. Through numerical calculation, the varying regularity of optimal phase angle of the system vibration characteristics is obtained, and the influence of the gear ratio on the vibration characteristics of the multi-stage gear transmission is revealed. A reliable theoretical basis can be provided for the high-quality design, shock absorption, and noise reduction research of the multi-stage gear transmission.

\section{Phase configuration of multi-stage gear transmission}

The phase configuration describes the phase state space of the gear meshing in the multi-stage gear transmission system. In order to describe the phase space, the parameters that affect the phase state are analyzed.

In a single stage gear pair, The alternate meshing of single and double teeth causes fluctuations of meshing stiffness $k(t)$ [23]. For spur gears the number of teeth in contact fluctuates between 1 and 2 , and its percentage is measured by the contact radio $\varepsilon$. At the same time there are certain differences in the meshing stiffness at different positions in the gearing process. It can be shown experimentally that the definition of meshing stiffness as a periodic rectangular wave function based on the contact radio $\varepsilon$ is well suited to the study of both the main and harmonic response of gears[24].

In a multi-stage transmission, the meshing stiffness of each stage is basically similar to the meshing stiffness of a pair of teeth. Taking a two-stage parallel shaft spur gear transmission system as an example, the coupling stiffness of the first stage and the second stage is the system stiffness. The meshing stiffness at each stage is approximately a periodic rectangular wave[25]. $k(t)$ to fluctuate roughly between $k$ and $2 k$ where $k t$ is the stiffness of a single tooth pair in contact. In the process of coupling, it may be in a single-tooth meshing state, a double-tooth meshing state, and one stage in a single-tooth meshing state and the other in a multi-tooth meshing state. Figure 1 shows the shapes of two rectangular waves with different periods. The meshing stiffness period of the first-stage gear is 
$T_{1}$, and the meshing stiffness period of the two-stage gear is $T_{2}$. Since the number of teeth is a positive integer, according to the coupling phase diagram in Figure 1, the period of system stiffness can be obtained as the least common multiple relationship of $T_{1}$ and $T_{2}$. There is a time-independent phase difference between the two meshing stiffnesses, and different phase differences correspond to different coupling stiffnesses of the system. According to the cd in Figure 1, it can be concluded that the two coupling stiffnesses are the same under time-independent conditions, and the period of their difference is $\Psi_{12}$.
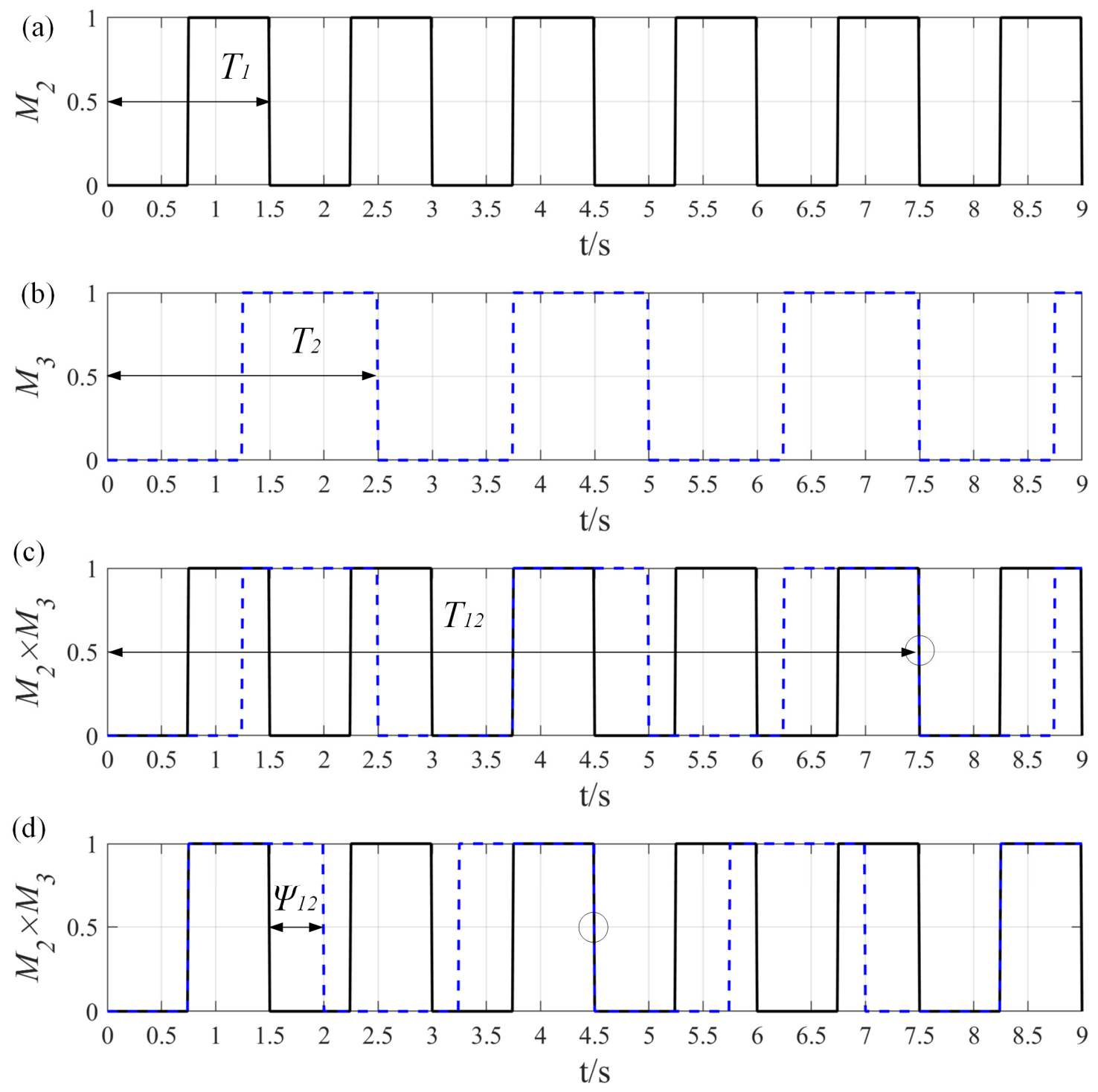

Fig. 1 The state image of periodic function

According to Fig. 1, the phase is reflected in the phase difference between the two gears of the intermediate shaft. According to the meshing principle of the gear transmission, this paper derives the minimum phase difference relationship. First making mathematical assumptions about the problem. It is assumed that the dynamic characteristics of each gear are the same, so that the machining error of the tooth profile of each gear is not considered; It is assumed that the pitch is equal, so that the position deviation between teeth is not considered.

Suppose $\mathbf{M}$ is a single pair of gear meshing space. It is considered that this space can contain 
all but the meshing conditions of the gears. Based on the assumption of the gear transmission system, it can be obtained that the meshing space of a single pair of teeth is complete in the meshing cycle, that is, it can describe the meshing state at any time. $M_{i}$ is the meshing state of a pair of gears determined in the meshing space $\mathbf{M}$, where $M_{i} \in \mathbf{M}$. Based on the nature of the periodic function, the function Eq. (1) of the fitted state about time and period is given.

$$
M_{i}=f_{i}\left(t, T_{i}\right)=f_{i}\left(t+T_{i}, T_{i}\right)
$$

where, $t$ is the time variable, and $T_{i}$ is the meshing period, which is related to the speed and the number of teeth. Based on the meshing space of a single pair of gears, the meshing space of the multi-stage gear transmission system is derived. Taking the two-stage gear transmission system as an example, the meshing state of the first-stage gear transmission is set as $M_{1}$, the meshing state of the second-stage gear transmission is set as $M_{2}$, Then the meshing state of the secondary gear transmission system is $M_{1} \times M_{2}$. According to Eq.(1),

$$
\begin{gathered}
\left\{\begin{array}{l}
M_{1}=f_{1}\left(t_{1}, T_{1}\right) \\
M_{2}=f_{2}\left(t_{2}, T_{2}\right)
\end{array}\right. \\
M_{1} \times M_{2}=f_{1}\left(t_{1}, T_{1}\right) \times f_{2}\left(t_{2}, T_{2}\right)
\end{gathered}
$$

The physical meaning of $M_{1} \times M_{2}$ is the coupling between the meshing state of the first stage and the meshing state of the second stage. Next, $f_{1}(\cdot) \times f_{2}(\cdot)$ needs to be derived based on analysis and derivation. $f_{1}(\cdot) \times f_{2}(\cdot)$

The meshing state of the two-stage gear transmission system has a phase difference $\varphi_{12}=\varphi_{1}-\varphi_{2}$ in the coupling process of the single-stage meshing state. The phase difference does not depend on time. It represents the deflection angle between the meshing position between the first and the second stage, and is adjusted by the two gears of the intermediate shaft. In order to describe the two-stage gear transmission system completely, the phase difference $\varphi$ needs to be continuously changed within the minimum phase difference period. It can be obtained that the minimum phase difference period is an important parameter to describe the meshing state of the two-stage gear transmission system, and then the minimum phase difference period of the two-stage gear transmission system is derived.

According to the speed of the intermediate shaft, the meshing period of the first stage and the second stage is obtained.

$$
\left\{\begin{array}{l}
T_{1}=\frac{1}{n_{1} z_{1}}=\frac{1}{n_{2} z_{2}} \\
T_{2}=\frac{1}{n_{3} z_{3}}=\frac{1}{n_{4} z_{4}}
\end{array}\right.
$$

Where, $n_{j}$ represents the speed of the $j$-th gear, $z_{j}$ represents the number of teeth of the $j$-th gear, $\mathrm{j}$ (odd integer) and $j+1$ are meshing gears of the same stage. Since the speeds of the two middle gears are the same $n_{2}=n_{3}$, the meshing period $T_{1}$ and $T_{2}$ are related to the number of teeth $z_{2}$ and $z_{3}$ of the two gears. The total period of the two-stage gear system:

$$
T_{12}=\frac{\left[z_{2}, z_{3}\right]}{n_{2} z_{2} z_{3}}
$$

$[\cdot, \cdot]$ is the symbol for finding the least common multiple of two integers. Since the number of teeth is a positive integer, when the two periodic functions are coupled, there is a situation that the 
meshing space is not full in the phase state space. Calculating the minimum phase difference can form a complete mesh phasing space of the two-stage drive. The minimum phase difference is the resolution of the number of teeth of the intermediate gear. The resolution of gear 2 is $z_{3} /\left(z_{2}, z_{3}\right)$, and the resolution of gear 3 is $z_{2} /\left(z_{2}, z_{3}\right)$ in the same way. Practical application is that the minimum phase difference can be adjusted for gear 2 or gear 3 .

The adjusted maximum deflection angle is for gear 2 with a single tooth corresponding to $\Phi_{1}=\left(z_{2}, z_{3}\right) / z_{3}$ of center angle or gear 3 with a single tooth corresponding to $\Phi_{2}=\left(z_{2}, z_{3}\right) / z_{2}$ of center angle, and the absolute corner is the same. According to Eqs. (2)-(5), derived from the above:

$$
\Phi_{12}=\frac{\left(z_{2}, z_{3}\right)}{z_{2} z_{3}}
$$

Where, $(\cdot, \cdot)$ is the symbol for finding the greatest common divisor of two integers. After the minimum phase difference is obtained, the meshing state of the two-stage gear transmission system is deduced to obtain:

$$
M_{1} \times M_{2}=f_{12}\left(t, \varphi, T_{12}, \Phi_{12}\right)
$$

Where, the coupling state of the two-stage gear transmission is defined as a function, and there are free variables $t$ and $\varphi . T_{12}$ is the periodic characteristic quantity that characterizes respect the coupling stage. $\Phi_{12}$ represents the space period characteristic quantity of the coupling state. When changing an integer multiple of $\Phi_{12}$, there is a certain gap in its state at time $t$, which can be clearly seen from Figure 1(c)(d). For a gear system with smooth gear movement, although $\Delta t$ exists, it is considered to be the same system. Eq. (8) represents the periodic characteristic of the coupled state about $T_{12}$ and $\Phi_{12}$.

$$
\left\{\begin{array}{l}
f_{12}\left(t+T_{12}, \varphi, T_{12}, \Phi_{12}\right)=f_{12}\left(t, \varphi, T_{12}, \Phi_{12}\right) \\
f_{12}\left(t, \varphi+\Phi_{12}, T_{12}, \Phi_{12}\right)=f_{12}\left(t+\Delta t, \varphi, T_{12}, \Phi_{12}\right)
\end{array}\right.
$$

The meshing space is derived into any stage gear transmission system. Definition $\mathbf{M}^{n}$ is the meshing space of the n-stage gear transmission system, and $M^{n}$ is the meshing state of the n-stage gear transmission system with given parameters.

$$
M^{n}=\left(M_{1} \times M_{2}\right)\left(M_{2} \times M_{3}\right) \cdots\left(M_{i} \times M_{i+1}\right) \cdots\left(M_{n-1} \times M_{n}\right)
$$

where, $M_{i} \times M_{i+1}=f_{i(i+1)}\left(t, \varphi, T_{i(i+1)}, \Phi_{i(i+1)}\right)$, In n-stage transmission, the coupling between every two stages can be constructed with the Eq.(7). For the coupling state of n-stage, the gear ratio between the couplings needs to be considered. $t$ is a common quantity, and $\varphi$ is independent variable between each two stages. The meshing state space representation of any two stages is obtained, which is deduced into the n-stage gear transmission system. It can be obtained that the meshing state space of the n-stage gear transmission system is:

$$
M^{n}=f^{n}\left(t, \varphi_{1}, \varphi_{2}, \cdots, \varphi_{n 1}, T^{(n)}, \Phi^{[1]}, \Phi^{[2]}, \cdots, \Phi^{[n-1]}\right)
$$

Where, $t$ is a continuously changing time variable. $\varphi_{i}$ represents the phase difference between $i$ and $i+1$ stage gear drives, and its maximum value is $\Phi^{[i]}$.

$$
\Phi^{[i]}=\Phi_{i(i+1)}=\left(z_{2 i-2}, z_{2 i-1}\right) / z_{2 i-2} z_{2 i-1}
$$

$T^{(n)}$ is the period of meshing state space of n-stage gear transmission system. The derivation is Eqs. (11)-(13):

$$
\begin{gathered}
T^{(n)}=T_{12 \cdots n}=\left[T_{1}, T_{2}, T_{3}, \cdots, T_{n}\right] \\
T^{(n)}=\left[T_{1}, T_{2}, T_{3}, \cdots, T_{n}\right]
\end{gathered}
$$


In order to facilitate calculation, the meshing state space of n-stage gear transmission system is defined recursively.

$$
\left\{\begin{array}{l}
T^{(n)}=\left[T^{n-1}, T_{n}\right] \\
T^{(1)}=T_{1}
\end{array}\right.
$$

Where, $T_{n}=\frac{z_{4} z_{6} \cdots z_{2 n-2}}{n_{2} z_{3} z_{5} \cdots z_{2 n-1}}$.

\section{Dynamic model of two-stage gear transmission}

The gear ratio of each stage, the tooth number ratio of two gears of the intermediate shaft, and their phase angle leads to the difference of vibration characteristics in multistage gear transmission. It is necessary to study the influence of phase configuration on the vibration characteristics of the multistage gear transmission system.

For describing to nonlinear dynamic behavior in a two-stage gear transmission, dynamic model containing the intermediate shaft is considered in Figure 2. Among these are the basic parameters of the gear system, including the inertia $J_{i}(i=1,2,3,4)$ of the four gears and the number of teeth $Z_{i}$ $(i=1,2,3,4)$. Input torque $T_{1}$ and output torque $T_{2}$ for stable operation of the system. The dynamic parameters of the gear are mainly considered in terms of time-varying meshing stiffness $k_{12}(t)$ and $k_{34}(t)$, meshing damping $c_{12}$ and $c_{34}$, and non-linear clearance $2 b$. the intermediate shaft considering its torsional stiffness $k_{\alpha}$ and torsional damping $c_{\alpha}$. The equations of motion of this six-degree-offreedom (4-DOF) system are written as Eq. (14).

$$
\left\{\begin{array}{l}
J_{1} \ddot{\theta}_{1}+r_{b 1}\left[c_{12}\left(r_{b 1} \dot{\theta}_{1}-r_{b 2} \dot{\theta}_{2}\right)+k_{12}(t) f\left(r_{b 1} \theta_{1}-r_{b 2} \theta_{2}\right)\right]=T_{1} \\
J_{2} \ddot{\theta}_{2}-r_{b 2}\left[c_{12}\left(r_{b 1} \dot{\theta}_{1}-r_{b 2} \dot{\theta}_{2}\right)+k_{12}(t) f\left(r_{b 1} \theta_{1}-r_{b 2} \theta_{2}\right)\right]+c_{\alpha}\left(\dot{\theta}_{2}-\dot{\theta}_{3}\right)+k_{\alpha}\left(\theta_{2}-\theta_{3}\right)=0 \\
J_{3} \ddot{\theta}_{3}+r_{b 3}\left[c_{34}\left(r_{b 3} \dot{\theta}_{3}-r_{b 4} \dot{\theta}_{4}\right)+k_{34}(t) f\left(r_{b 3} \theta_{3}-r_{b 4} \theta_{4}\right)\right]-\left[c_{\alpha}\left(\dot{\theta}_{2}-\dot{\theta}_{3}\right)+k_{\alpha}\left(\theta_{2}-\theta_{3}\right)\right]=0 \\
J_{4} \ddot{\theta}_{4}-r_{b 4}\left[c_{34}\left(r_{b 3} \dot{\theta}_{3}-r_{b 4} \dot{\theta}_{4}\right)+k_{34}(t) f\left(r_{b 3} \theta_{3}-r_{b 4} \theta_{4}\right)\right]=-T_{2}
\end{array}\right.
$$

where, $r_{b 1}, r_{b 2}, r_{b 3}$, and $r_{b 3}$ are base circle radii.

$$
\left\{\begin{array}{l}
c_{\alpha}=2 \delta_{T} \sqrt{k_{\alpha} \frac{J_{0} J_{1}}{J_{0}+J_{1}}} \\
c_{\beta}=2 \delta_{T} \sqrt{k_{\beta} \frac{J_{2} J_{3}}{J_{2}+J_{3}}} \\
c_{\gamma}=2 \delta_{T} \sqrt{k_{\gamma} \frac{J_{5} J_{4}}{J_{5}+J_{4}}}
\end{array}\right.
$$

where, $\delta_{T}$ is the torsional damping ratio of the material. Tests have shown that $\delta_{T}$ has a value in the range $0.005-0.075$. 


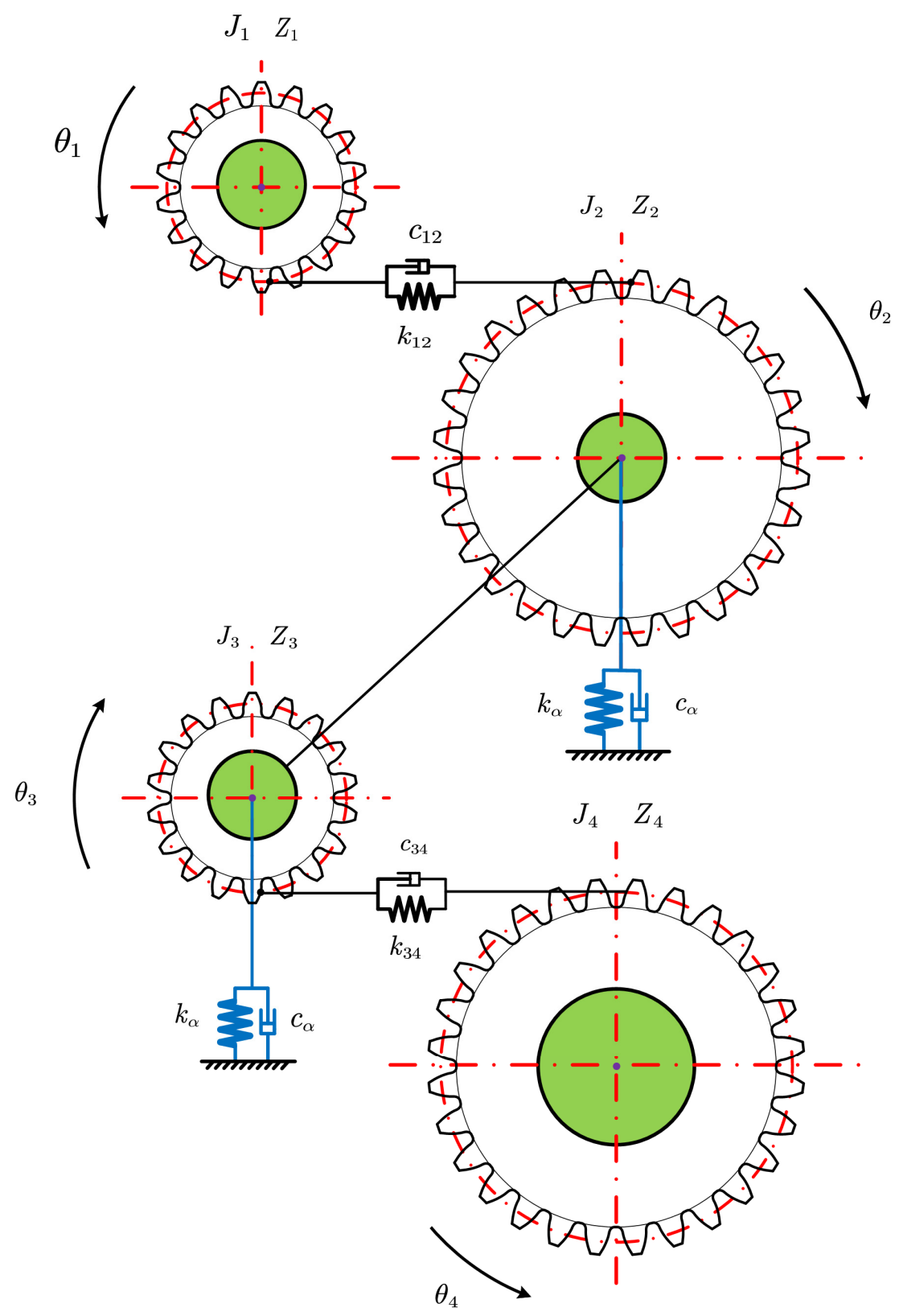

Fig. 2 Dynamic model of two-stage gear transmission system

To reduce computational complexity and accurately capture model characteristics, Eq. (14) is transformed into a 3-degree-of-freedom equation using Eq. (15).

$$
\left\{\begin{array}{l}
u_{1}=r_{b 1} \theta_{1}-r_{b 2} \theta_{2} \\
u_{2}=\frac{\left(r_{b 2}+r_{b 3}\right)}{2}\left(\theta_{2}-\theta_{3}\right) \\
u_{3}=r_{b 3} \theta_{3}-r_{b 4} \theta_{4}
\end{array}\right.
$$

$u_{1}$ represents the dynamic transmission error of the first stage gear, $u_{2}$ represents the torsional vibration characteristics of the intermediate shaft and $u_{2}$ represents the dynamic transmission error of the second stage gear. 


$$
\left\{\begin{array}{l}
m_{12} \ddot{u}_{1}+c_{12} \dot{u}_{1}+k_{1}(t) f\left(u_{1}\right)=m_{12} F_{1}+\alpha\left(c_{23} \dot{u}_{2}+k_{23} u_{2}\right) \\
m_{23} \ddot{u}_{2}+c_{23} \dot{u}_{2}+k_{23} u_{2}=\beta\left(c_{12} \dot{u}_{1}+k_{1}(t) f\left(u_{1}\right)\right)+\gamma\left(c_{34} \dot{u}_{3}+k_{34}(t) f\left(u_{3}\right)\right) \\
m_{34} \ddot{u}_{3}+c_{34} \dot{u}_{3}+k_{34}(t) f\left(u_{3}\right)=\varrho\left(c_{23} \dot{u}_{2}+k_{23} u_{2}\right)+m_{34} F_{2}
\end{array}\right.
$$

Where,

$$
\begin{aligned}
& m_{12}=\frac{J_{1} J_{2}}{J_{1} r_{b 2}^{2}+J_{2} r_{b 1}^{2}}, m_{23}=\frac{J_{2} J_{3}}{\left(J_{2}+J_{3}\right) r^{2}}, m_{34}=\frac{J_{3} J_{4}}{J_{3} r_{b 4}^{2}+J_{4} r_{b 3}^{2}}, r=\frac{r_{b 2}+r_{b 3}}{2}, \\
& c_{23}=\frac{c_{\alpha}}{r^{2}} k_{23}=\frac{k_{\alpha}}{r^{2}}, \alpha=\frac{r r_{b 2} m_{12}}{J_{2}}, \beta=\frac{r r_{b 2} m_{23}}{J_{2}}, \gamma=\frac{r r_{b 3} m_{23}}{J_{3}}, \varrho=\frac{r r_{b 3} m_{34}}{J_{3}}, \\
& F_{1}=\frac{r_{b 1}}{J_{1}} T_{1}, F_{2}=\frac{r_{b 4}}{J_{4}} T_{2} .
\end{aligned}
$$

Time-varying meshing stiffness is the main source of internal excitation. There are several common methods of calculating the meshing stiffness of gear pairs. In this paper, the equivalent tooth profile method will be used to solve the gear stiffness[26]. The single tooth stiffness is the reciprocal of the deformation of the teeth under unit external force. By the equivalent tooth profile method, the total flexibility of a single gear is calculated as follows:

$$
\delta_{i}=\delta_{F i}+\delta_{T i}+\delta_{G i}+\delta_{H i}=\delta_{F r i}+\delta+\delta_{T r i}+\delta_{T t i}+\delta_{G i}+\delta_{H i}
$$

Where, Total bending flexibility $\delta_{F i}$, Total shear flexibility $\delta_{T i}$, The total flexibility caused by the elastic tilt of the tooth base $\delta_{G i}$, Teeth contact flexibility $\delta_{H i}$.

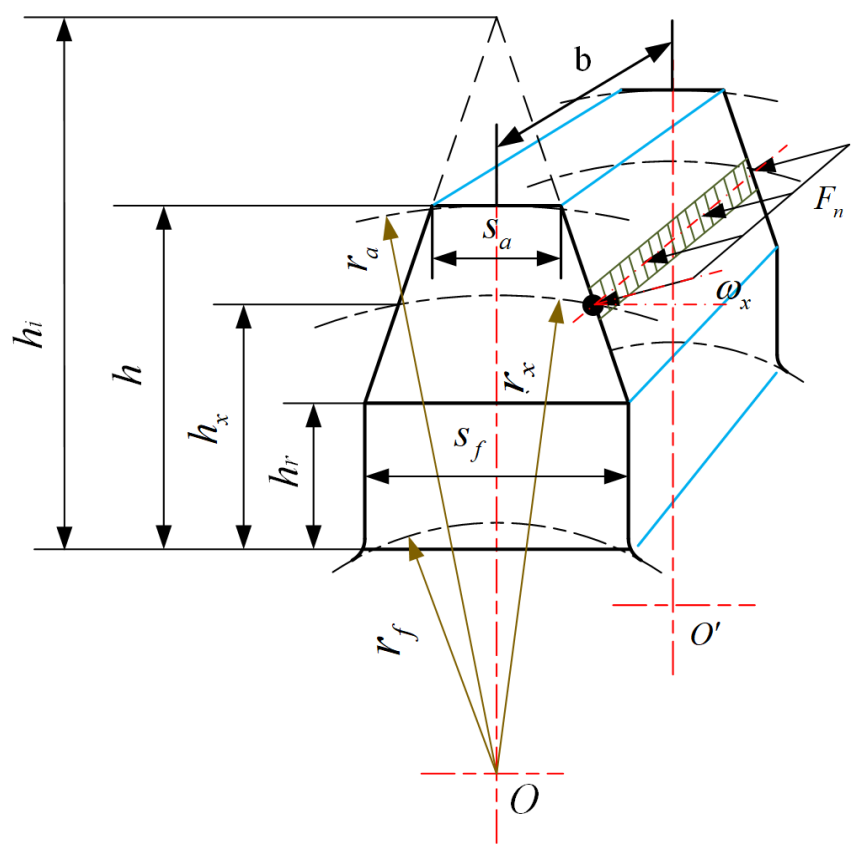

Fig. 3 Equivalent tooth profile geometric parameters

The total bending flexibility of the gear teeth is equivalent to the sum of the bending flexibility of the trapezoidal section and the bending flexibility of the rectangular section. The model established by the equivalent tooth profile method of gear teeth is shown in Figure 3. Through the approximate treatment of the involute gear profile, cuboid and trapezoid are used to replace the lower part of the tooth root and the upper part of the tooth tip respectively. Where $s_{a}$ is the width of the tooth tip, $s_{f}$ means the width of the tooth root, $r_{a}, r_{x}$ and $r_{f}$ are the tooth tip circle, index circle and tooth root circle radius respectively, $h, h_{x}, h_{f}$ and $h_{i}$ respectively indicate tooth height, meshing tooth height, tooth 
root height and auxiliary tooth height,$b$ refers to the width of the gear teeth, $\omega_{x}$ is the angle between the direction of the unit load and the horizontal axis, and $F_{n}$ is the linear load acting on the gear teeth. The calculation method is as follows:

$$
\delta_{F i}=\delta_{F r i}+\delta_{F t i}
$$

the bending flexibility of the trapezoidal section

$$
\delta_{F r i}=\frac{12 \cos ^{2} \omega_{x}}{E s_{f}^{3}}\left(h_{x}^{2} h_{r}-h_{x} h_{r}^{2}+\frac{h_{r}^{3}}{3}\right)
$$

the bending flexibility of the rectangular section

$$
\delta_{F t i}=\frac{6 \cos ^{2} \omega_{x}}{E s_{f}^{3}}\left(h_{i}-h_{r}\right)^{3}\left[\frac{h_{i}-h_{x}}{h_{i}-h_{r}}\left(4-\frac{h_{i}-h_{x}}{h_{i}-h_{r}}\right)-2 \ln \frac{h_{i}-h_{x}}{h_{i}-h_{r}}-3\right]
$$

The total shear compliance is calculated in the same way as the total flexion of the teeth, it can be expressed as a superposition of the partial flexibility and the trapezoidal partial flexibility:

$\delta_{T i}=\delta_{T r i}+\delta_{T t i}$

The shear deformation of the rectangular part.

$\delta_{T r i}=\frac{2(1+\nu) \cos ^{2} \omega_{x} h_{r}}{E s_{f}}$

The trapezoidal shear deformation.

$\delta_{T r i}=\frac{2(1+\nu) \cos ^{2} \omega_{x}\left(h_{i}-h_{r}\right)}{E s_{f}} \ln \frac{h_{i}-h_{r}}{h_{i}-h_{x}}$

The total flexibility caused by the elastic tilt of the tooth base $\varepsilon_{G i}$.

$\delta_{G i}=\frac{24 h_{x}^{2} \cos ^{2} \omega_{x}}{\pi E s_{f}^{3}}$

The monomer elastic variables are:

$\delta_{H i}=\frac{4 \rho}{\pi E \rho_{i}}$

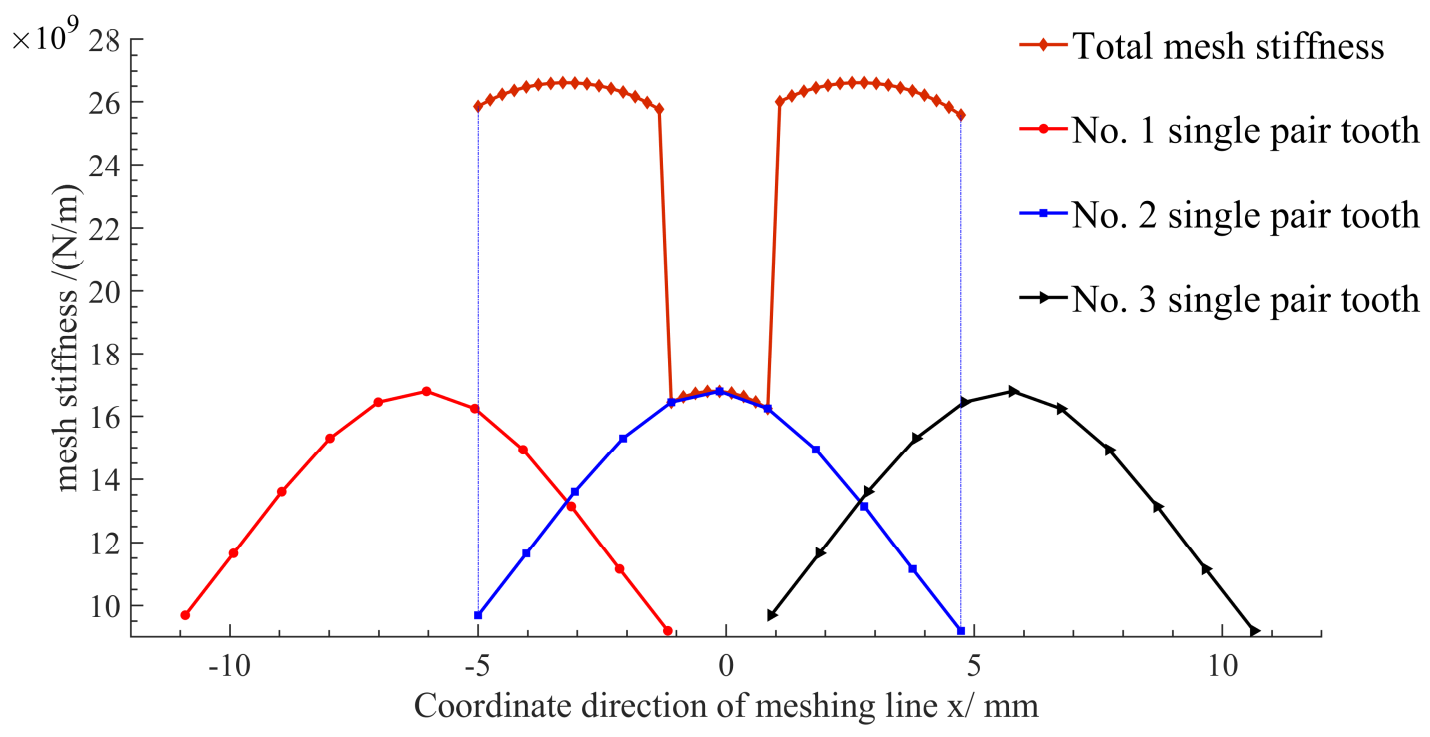

Fig. 4 Distribution of tooth meshing stiffness along line of action

Gear transmission is achieved by meshing teeth. For the meshing of the two gear teeth, the whole 
process of force deformation can be equivalent to the spring series contact model of two rigid gear teeth. The total amount of meshing available is:

$$
\delta_{s}=\delta_{1}+\delta_{2}
$$

The overall gear mesh stiffness is given by:

$$
\frac{1}{k_{s}}=\frac{1}{k_{1}}+\frac{1}{k_{2}}
$$

According to Eq. (17), the total meshing stiffness of the gear meshing process is obtained, as shown in Fig. 4.

When calculating the meshing stiffness of gears, there is stiffness fluctuation due to the change of the number of meshing teeth. For spur gears, the contact ratio is used to describe the proportion of single and double tooth meshing. The contact ratio generally adopts the ratio of meshing length to single tooth meshing length. In order to ensure the continuity of gear transmission, the contact ratio must be greater than 1 .

$$
\begin{gathered}
\varepsilon=\frac{\overline{B_{1} B_{2}}}{p_{b}} \\
\varepsilon=\frac{1}{2 \pi}\left[z_{1}\left(\tan \alpha_{a 1}-\tan \alpha^{\prime}\right)+z_{2}\left(\tan \alpha_{a 2}-\tan \alpha^{\prime}\right)\right]
\end{gathered}
$$

According to the Eq. the contact ratio is related to the number of teeth of the meshing gear. The tooth number pairs of different meshing gears will cause the different proportions of single and double tooth meshing areas.

Due to the existence of manufacturing error and assembly error, there will be a nonlinear backlash function between gear meshing. The nonlinear backlash function can be expressed as Eq.(20):

$$
f(x)= \begin{cases}x-b & \text { if } x>b \\ 0 & \text { if }-b<x<b \\ x+b & \text { if } x<-b .\end{cases}
$$

Dimensionless equation of motion can be obtained by defining:

$$
\begin{aligned}
& q_{i}=u_{i} / b, \omega_{n}=\sqrt{\bar{k}_{12} / m_{12}}, \tau=\omega_{n} t, \Omega=\omega_{k} / \omega_{n}, \\
& \zeta_{12}=r r_{b 2} c_{23} / J_{2} \omega_{n}, \kappa_{12}=r r_{b 2} k_{23} / J_{2} \omega_{n}^{2}, \zeta_{11}=r r_{b 2} c_{12} / J_{2} \omega_{n}, \\
& \kappa_{11}=r r_{b 2} k_{12} / J_{2} \omega_{n}^{2}, \zeta_{33}=r r_{b 3} c_{34} / J_{3} \omega_{n}, \kappa_{33}=r r_{b 3} k_{34} / J_{2} \omega_{n}^{2}, \\
& \zeta_{23}=r r_{b 3} c_{23} / J_{3} \omega_{n}, \kappa_{23}=r r_{b 3} k_{34} / J_{3} \omega_{n}^{2}, \zeta_{i}=c_{(2 i-1)(2 i)} / 2 m_{(2 i-1)(2 i)} \omega_{n}, \\
& \kappa_{i}=k_{(2 i-1)(2 i)} / m_{(2 i-1)(2 i)} \omega_{n}^{2}, \tilde{F}_{i}=F_{i} / b \omega_{n}^{2}
\end{aligned}
$$

The dimensionless equation of the gear pair could be written as:

$$
\left\{\begin{array}{l}
\ddot{q}_{1}+2 \zeta_{1} \dot{q}_{1}+\kappa_{1}(t) f\left(q_{1}\right)=F_{1}+\zeta_{12} \dot{q}_{2}+\kappa_{12} q_{2} \\
\ddot{q}_{2}+2 \zeta_{2} \dot{q}_{2}+\kappa_{2} q_{2}=\zeta_{11} \dot{q}_{1}+\kappa_{11} q_{1}+\zeta_{33} \dot{q}_{3}+\kappa_{33} q_{3} \\
\ddot{q}_{3}+2 \zeta_{3} \dot{q}_{3}+\kappa_{3}(t) f\left(q_{3}\right)=F_{2}+\zeta_{23} \dot{q}_{2}+\kappa_{23} q_{2}
\end{array}\right.
$$

\section{Example and discussions}

Based on the dynamic model of a two-stage gear transmission system, the corresponding parameters are calculated. Runge Kutta numerical method is used to solve the vibration displacement and vibration velocity. The parameter of the two-stage gear system is shown in Table. 1 . In order to explore the influence of the phase configuration on the vibration characteristics of the two-stage gear transmission system, three sets of gear ratio $\left(r_{1}=4, r_{2}=4.18, r_{3}=3.85\right)$ with similar total gear ratios 
are selected for comparison and analysis.

Table 1 Design parameters of the gear system

\begin{tabular}{cc}
\hline Parameters & Values \\
\hline Modulus $(m m)$ & 3 \\
The number of teeth at the gear ratio1 & $z_{1} 12 ; z_{2} 24 ; z_{3} 12 ; z_{4} 24$ \\
The number of teeth at the gear ratio2 & $z_{1} 12 ; z_{2} 25 ; z_{3} 13 ; z_{4} 24$ \\
The number of teeth at the gear ratio3 & $z_{1} 11 ; z_{2} 24 ; z_{3} 12 ; z_{4} 23$ \\
Tooth width $(m m)$ & 20 \\
Pressure angle $\left(^{\circ}\right)$ & 20 \\
Load torque $(N \cdot m)$ & 400 \\
\hline
\end{tabular}

the meshing state of the gear transmission system under three gear ratios is analyzed based on the phase configuration. According to Eq.(7), Derive the coupling state of the two-stage gear system $M^{2}=f^{2}\left(t, \varphi, T^{(2)}, \Phi^{[2]}\right)$, Calculate $T^{(2)}$ and $\Phi^{[2]}, T_{1}^{(2)}=1 / 12 n_{2}, T_{2}^{(2)}=1 / n_{2}, T_{2}^{(2)}=1 / 12 n_{2}$ ， $\Phi_{1}^{[2]}=1 / 24, \Phi_{2}^{[2]}=1 / 325, \Phi_{3}^{[2]}=1 / 24$. The $T^{(2)}$ and $\Phi^{[2]}$ of gear ratio 1 and gear ratio 3 are equal, they have the same periodicity, but contact ratios are different because of the difference of $z_{1}$ and $z_{4}$. the proportions of $p k$ and $(p+1) k$ are different. The period of gear ratio 1 is lower than that of gear ratio 2 , which means that the frequency of gear ratio 1 meshing error will be higher than gear ratio 2 . The $\Phi^{[2]}$ of gear ratio 1 is greater than gear ratio 2, and the adjustment range of the deviation angle of the intermediate shaft of gear ratio 1 is smaller than that of gear ratio 2 . In the numerical calculation process, the phase of a single tooth relative to the third tooth is used, $\varphi_{1}^{[2]}=1 / 2, \varphi_{2}^{[2]}=1 / 25, \varphi_{3}^{[2]}=1 / 2$. In order to eliminate the influence of the initial state, one must search the entire initial conditions map.

For a two-stage gear transmission system with a gear ratio 1 of $z_{1} 12 ; z_{2} 24 ; z_{3} 12 ; z_{4} 24$ and dimensionless mesh frequency $\Omega_{1}=0.326$, the influence of the phase angle on its vibration characteristics is explored through numerical calculations (Fig. 5). The tooth ratio of the intermediate shaft is $12 / 24$, the minimum dimensionless phase angle $\varphi_{1}^{[2]}=1 / 2$, and the range of phase angle is large. In Figure 5a, $\dot{q}_{1}$ is the dimensionless vibration velocity, mesh cycle represents the number of meshing stiffness cycles, and phase is the difference $\left(\varphi_{1}^{[2]}=1 / 2\right)$ in the mesh phasing between the first and second stages.

It can be seen from Fig. 5 that there is the optimal phase angle to make the vibration amplitude of the system smaller, where the optimal phase angle can be obtained through the isogram $\varphi_{1}^{[2]}=0,0.2,0.35,0.5$. It can be obtained that there are 3 optimal phase angles at $\Omega_{1}=0.3$ to reduce the vibration amplitude of the system so as to achieve the effect of vibration reduction and noise reduction. Based on the phase configuration theory, the meshing state space of a two-stage gear transmission system is deduced, and its optimal phase angle is obtained. 


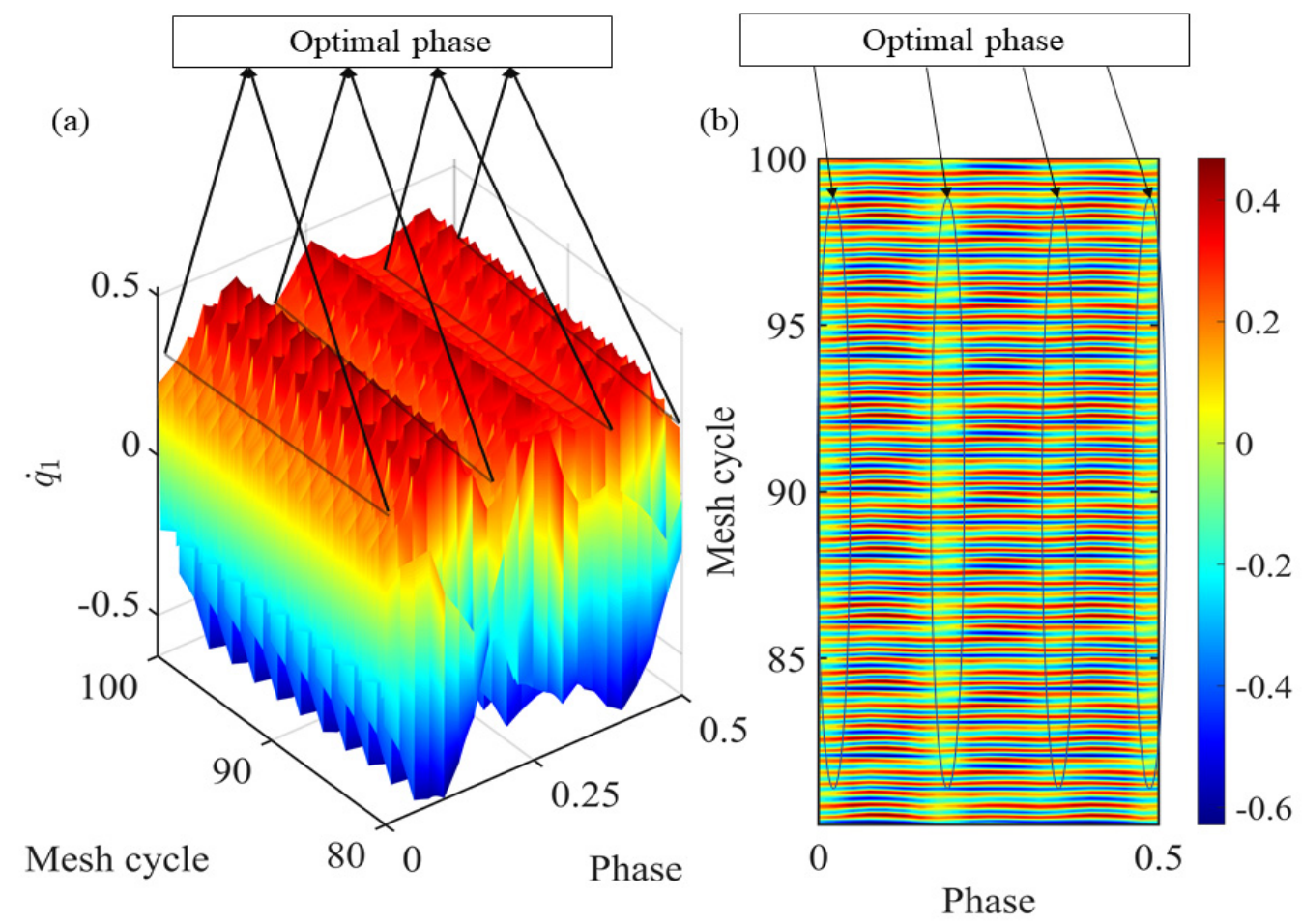

Fig. 5 Effect of phase on vibration characteristics

In order to reveal the Varying regularity of the optimal phase angle of the two-stage gear system by considering the phase configuration is considered, the vibration characteristic curves at different mesh frequency and phases are solved. The calculated root-mean-square (RMS) result of the gear ratio 1 of $\dot{q}_{1}$ is shown in Figure 6 .

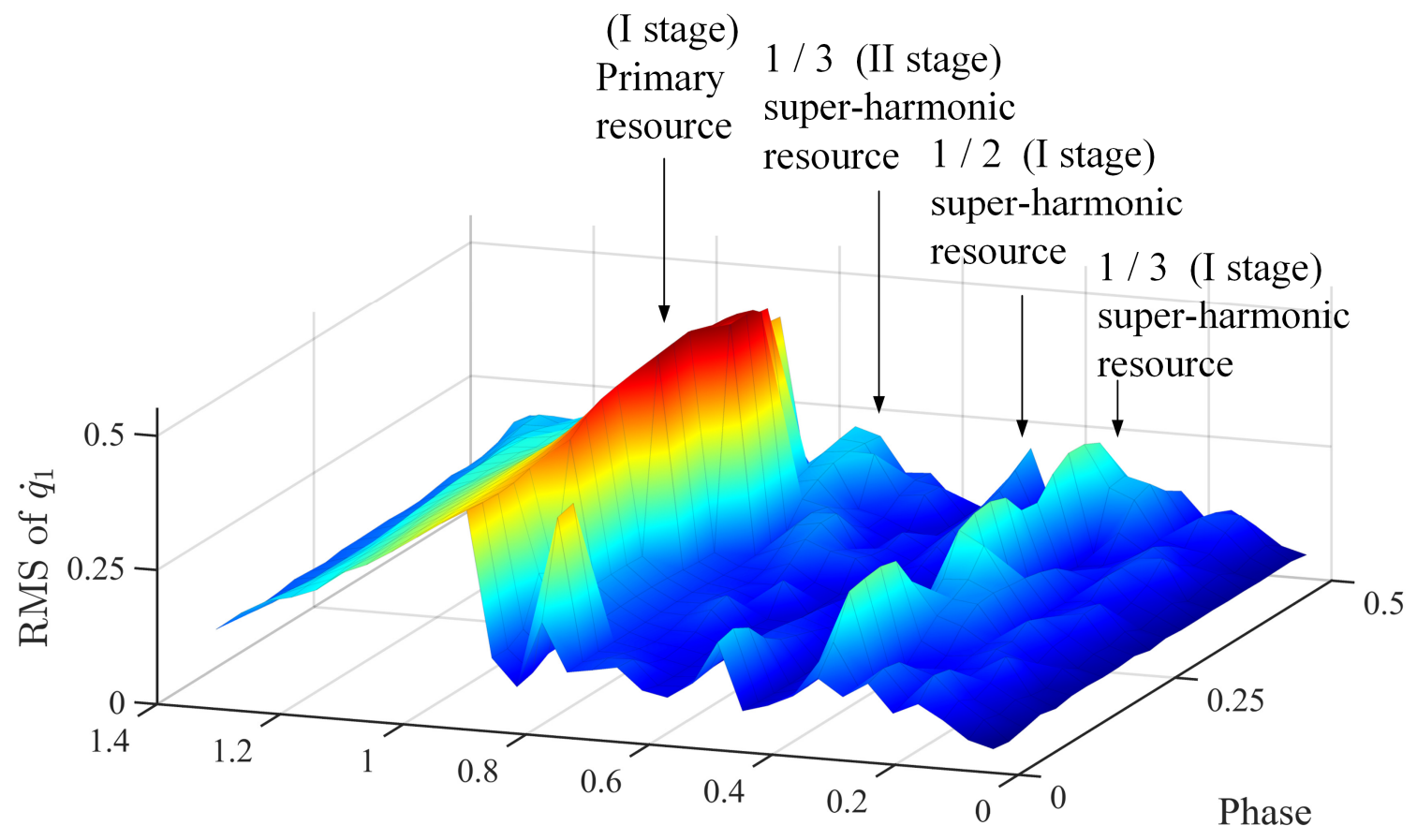

Mesh Frequency of $\Omega_{1}$

Fig. 6 The RMS of $\dot{q}_{1}$ of gear ratio 1 
Fig. 6 shows that varying regularity of optimal phase angle with mesh frequency at a gear ratio 1 . Three resonances of the first stage and one resonance of the second stage can be clearly obtained. Between the mesh frequency at the primary resonance of the I stage and at the $1 / 3$ super-harmonics resonance of the II stage, The system has a non-linear jump in the region, which is defined by a softening-type curve. This behavior usually occurs due to single-sided impact (SSI) motions of the gear. In addition, the amplitude of the $1 / 3$ super-harmonics resonance of the I stage, the $1 / 2$ superharmonics resonance of the I stage, and the 1/3 super-harmonics resonance of the II stage can be significantly reduced by the phase. This is due to the proportion of harmonic components that are changed by the phase. Below the mesh frequency at the 1/3 super-harmonics resonance of the I stage, the number of optimal phase angles decreases as the mesh frequency increases. Between the mesh frequency at the $1 / 2$ super-harmonics resonance of the I stage and the mesh frequency at the $1 / 3$ superharmonics resonance of the I stage, here the number of optimal phase angle continues to decrease as the frequency of engagement increases and there only one optimal phase angle at the $1 / 2$ superharmonics resonance of the I stage. Between the mesh frequency at the $1 / 3$ super-harmonics resonance of the II stage and at the 1/2 super-harmonics resonance of the I stage, the number of optimal phase angles increases in jumps then decreases as the mesh frequency.
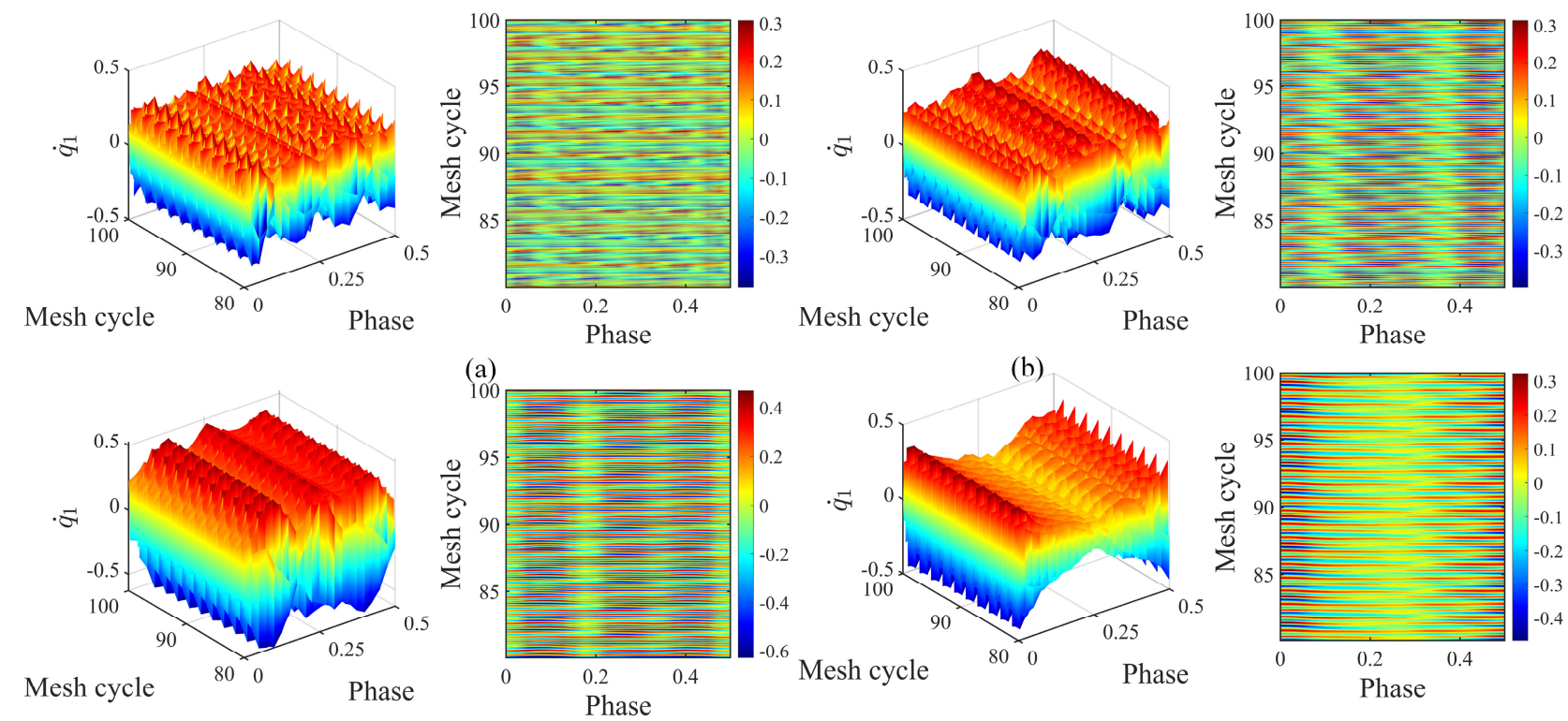

(b)

(c)
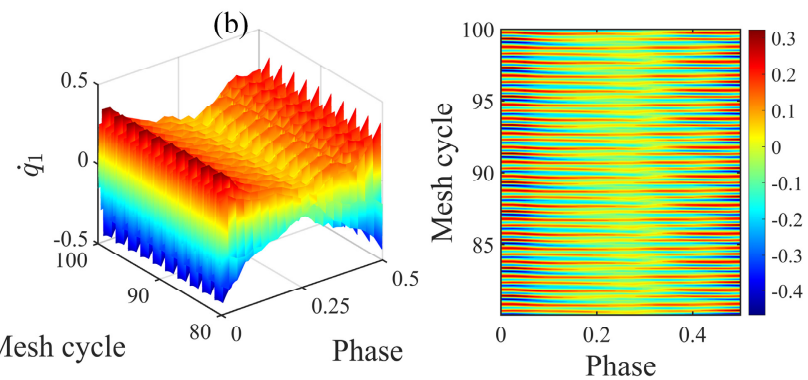

(d)
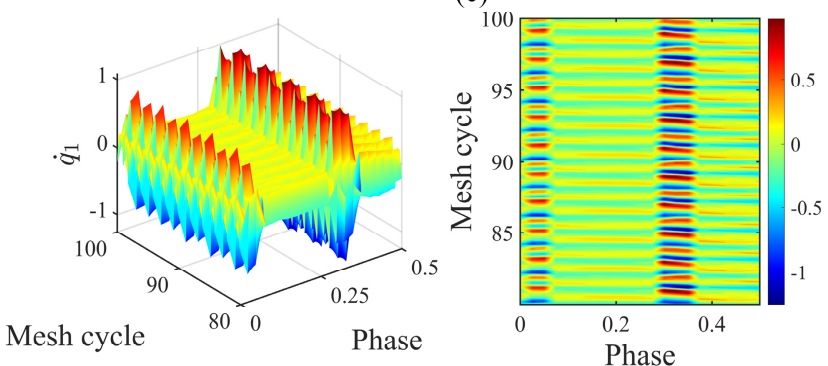

(e)

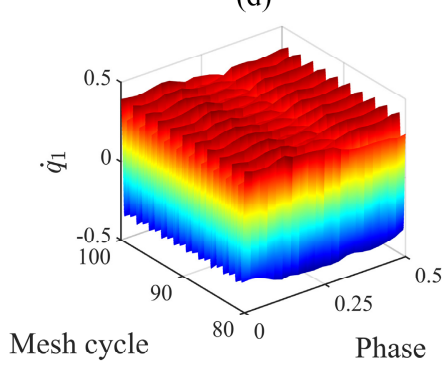

(f)

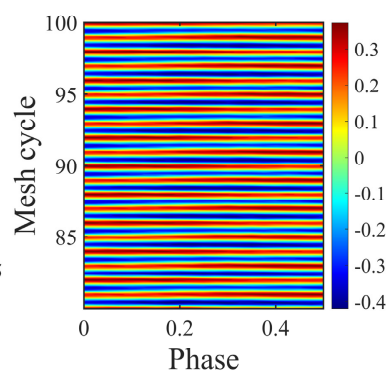

Fig. 7 Effect of phase Configuration on Vibration Curve at different mesh frequency of gear ratio 1
(a) $\Omega_{1}=0.15$
(b) $\Omega_{1}=0.25$
(c) $\Omega_{1}=0.30$
(d) $\Omega_{1}=0.5$
(e) $\Omega_{1}=0.75$
(f) $\Omega_{1}=1.15$

For obtain the accurate and optimal phase angle distribution, the vibration curve of different phase angles between $\Omega_{1}=0.05$ and $\Omega_{1}=1.4$ is calculated with the mesh frequency of $\Omega_{1}=0.05$ as the interval. Some of these results are shown in Fig. 7. It is obvious that there are 4 optimal phase angles at $\Omega_{1}=0.15,2$ optimal phase angles at $\Omega_{1}=0.25,3$ optimal phase angles at $\Omega_{1}=0.3,1$ 
optimal phase at $\Omega_{1}=0.5$. The optimal phase angle is not obvious at $\Omega_{1}=1.15$, and it may be near the one at $\varphi_{1}^{[2]}=0.27$. At $\Omega_{1}=0.75$, the system is in the non-linear jump region and the change in phase gives the gears no impact and single-sided impact motion. The varying regularity of optimal phase angle obtained from the vibration curve is basically consistent with that in Fig. 6 . Fast Fourier transform (FFT) of the data was taken after the application of a Hanning window to data to convert time-domain data into the frequency domain. The spectrum result is shown in Fig. 8. When $\Omega_{1}=0.30$, the optimal phase angle is $\varphi_{1}^{[2]}=0,0.175,0.35$. When $\Omega_{1}=1.15$, the optimal phase angle is calculated as $\varphi_{1}^{[2]}=0.1$.

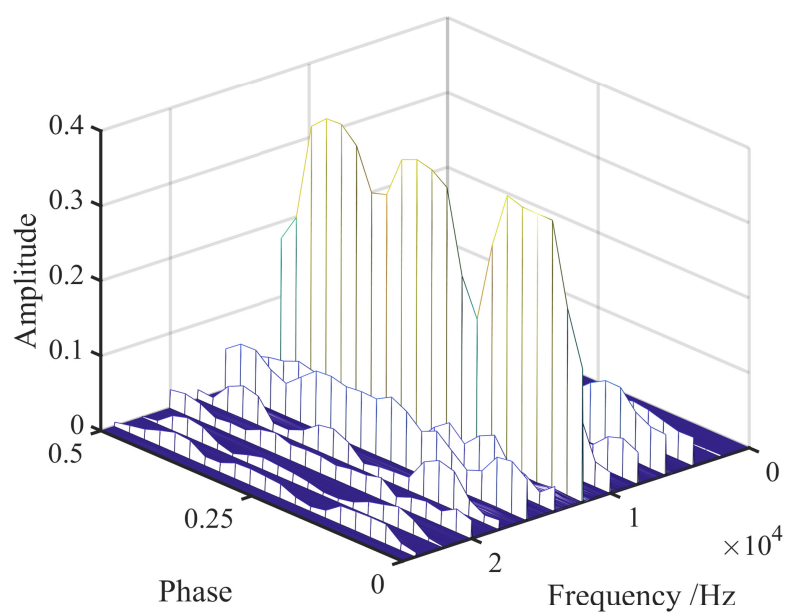

(a)

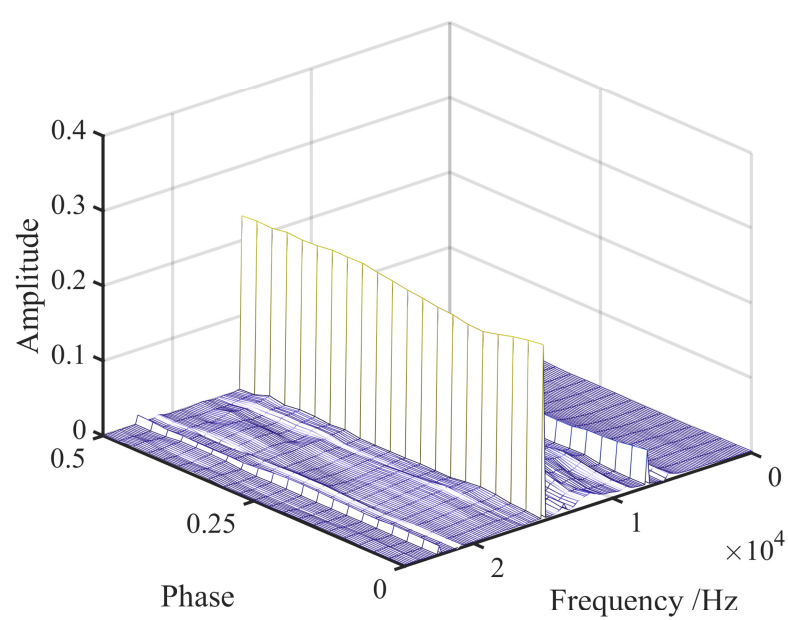

(b)

Fig. 8 FFT spectrum of phase configuration at different mesh frequency gear ratio 1 (a) $\Omega_{1}=0.30$

(b) $\Omega_{1}=1.15$

According to the theory of phase configuration, the tooth ratio of the intermediate shaft has a greater influence on the range of phase angle. This section explores the vibration characteristics and the optimal phase angle varying regularity under different gear ratios.

For gear ratio 2 of $z_{1} 12 ; z_{2} 25 ; z_{3} \quad 13 ; z_{4} 24$ and gear ratio 3 of $z_{1} 11 ; z_{2} 24 ; z_{3} 12 ; z_{4} 23$, the influence of phase angle on vibration characteristics under different mesh frequency conditions is explored. The calculated root-mean-square (RMS) result of the gear ratio 2 of $\dot{q}_{1}$ is shown in Figure 9. Similar to gear ratio 1, Fig. 9 shows that varying regularity of optimal phase angle with mesh frequency at a gear ratio 2 . It can be quite obvious that the primary resonance of the I stage, the $1 / 3$ super-harmonics resonance of the II stage, the 1/2 super-harmonics resonance of the I stage, and the $1 / 3$ super-harmonics resonance of the I stage The optimal phase angle is not obvious and the phase still has no effect on the vibration characteristics. 


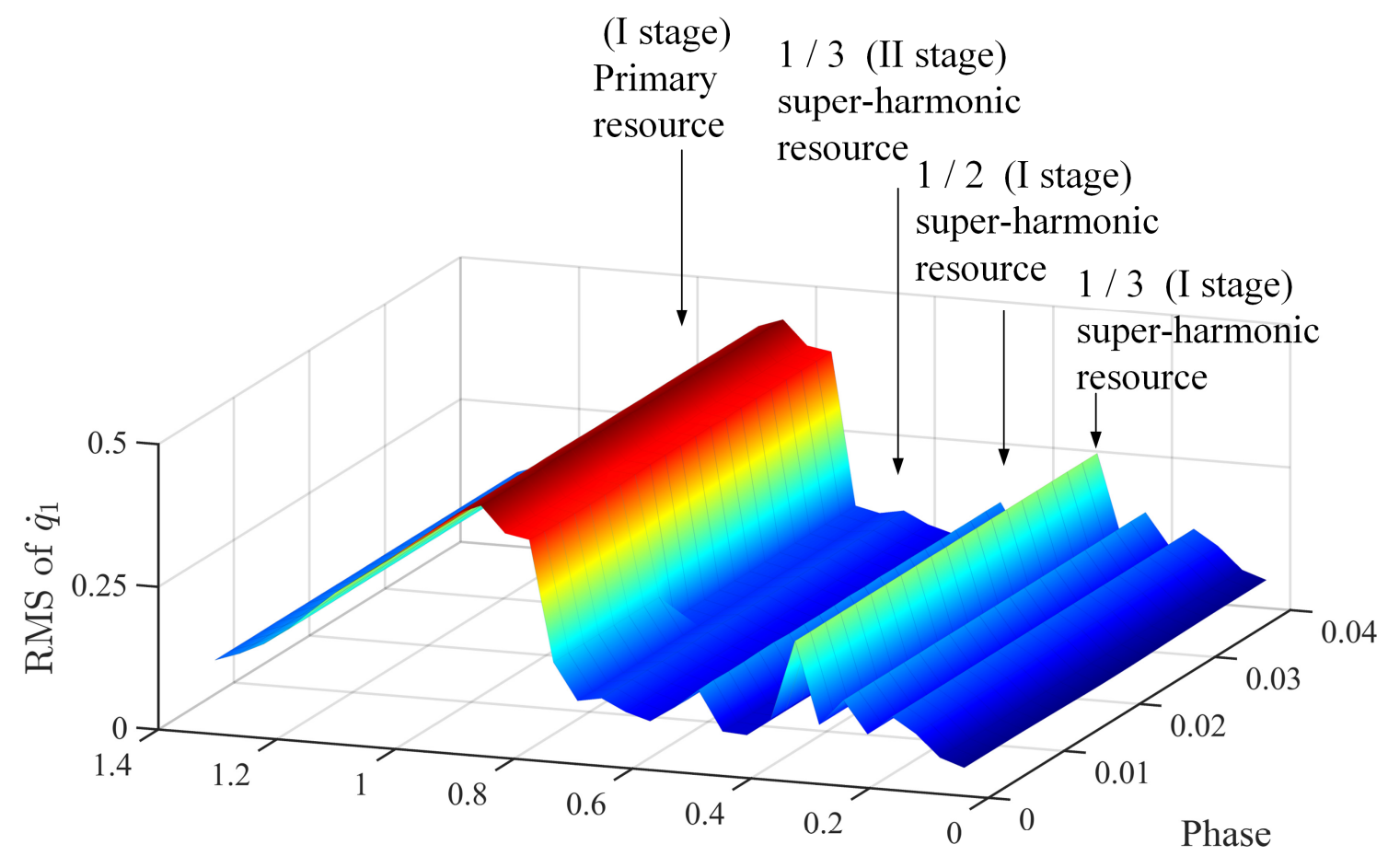

Mesh Frequency of $\Omega_{1}$

Fig. 9 The RMS of $\dot{q}_{1}$ of gear ratio 2

Table 2 Phase parameters of gear ratiol and gear ratio 2

\begin{tabular}{|c|c|c|}
\hline Parameters & Gear ratio1 & Gear ratio 2 \\
\hline Gear ratio & $z_{1} 12 ; z_{2} 24 ; z_{3} 12 ; z_{4} 24$ & $z_{1} 12 ; z_{2} 25 ; z_{3} 13 ; z_{4} 24$ \\
\hline Dimensionless phase $\varphi^{[2]}$ & 0.5 & 0.04 \\
\hline Periodicity $T^{(2)}$ & $1 / 12 n_{2}$ & $1 / n_{2}$ \\
\hline Contact ratio $\varepsilon$ & $\varepsilon_{1} 1.5111, \varepsilon_{2} 1.5111$ & $\varepsilon_{1} 1.5160, \varepsilon_{2} 1.5222$ \\
\hline
\end{tabular}

The varying regularity of optimal phase angle for gear ratio 1 and gear ratio 2 are compared and analyzed. The relevant parameters are shown in Table 2. $\varphi_{2}^{[2]}$ less than $\varphi_{1}^{[2]}$ means that the phase shift range is smaller of gear ratio 2, while its system period is longer, the phase critical quarters are lower, and the phase effect on vibration is lower.

The calculated root-mean-square (RMS) result of the gear ratio 3 of $\dot{q}_{1}$ is shown in Figure 10 . Three resonances of the first stage and one resonance of the second stage can be clearly obtained. Between the mesh frequency at the primary resonance of the I stage and at the 1/3 super-harmonics resonance of the II stage, The system has a non-linear jump in the region, which is defined by a softening-type curve. This behavior usually occurs due to single-sided impact (SSI) motions of the gear. In addition, the amplitude of the 1/3 super-harmonics resonance of the I stage, the $1 / 2$ superharmonics resonance of the I stage, and the 1/3 super-harmonics resonance of the II stage can be significantly reduced by the phase. This is due to the proportion of harmonic components that are 
changed by the phase. Below the mesh frequency at the $1 / 3$ super-harmonics resonance of the I stage, the number of optimal phase angles decreases as the mesh frequency increases. Between the mesh frequency at the $1 / 2$ super-harmonics resonance of the I stage and the mesh frequency at the $1 / 3$ superharmonics resonance of the I stage, here the number of optimal phase angle continues to decrease as the frequency of engagement increases and there only one optimal phase angle at the $1 / 2$ superharmonics resonance of the I stage. Between the mesh frequency at the $1 / 3$ super-harmonics resonance of the II stage and at the 1/2 super-harmonics resonance of the I stage, the number of optimal phase angles increases in jumps then decreases as the mesh frequency.

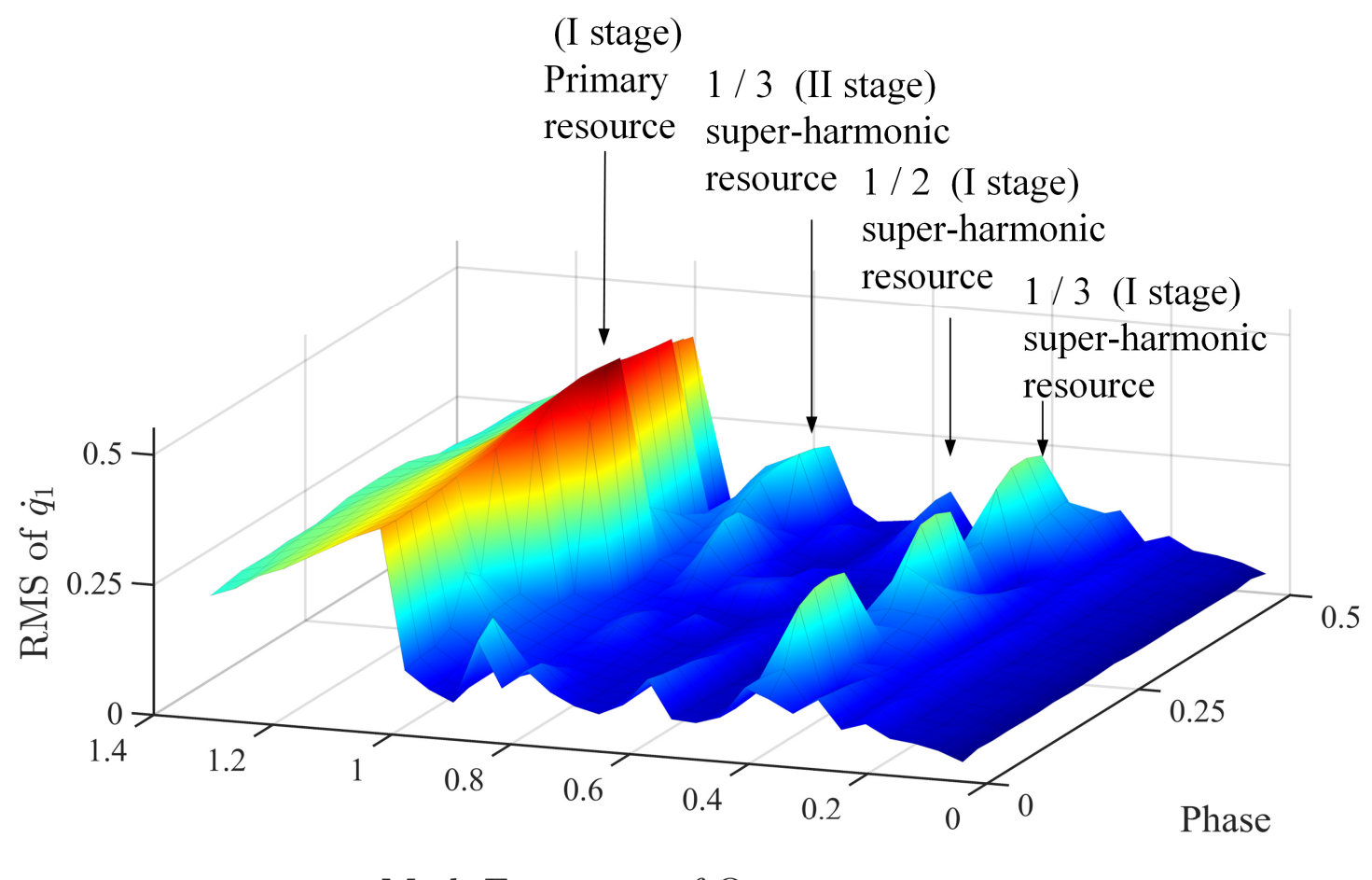

Mesh Frequency of $\Omega_{1}$

Fig. 10 The RMS of $\dot{q}_{1}$ of gear ratio 3

Table 3 Phase parameters of gear ratio1 and gear ratio 3

\begin{tabular}{|c|c|c|}
\hline Parameters & Gear ratio1 & Gear ratio 3 \\
\hline Gear ratio & $z_{1} 12 ; z_{2} 24 ; z_{3} 12 ; z_{4} 24$ & $z_{1} 11 ; z_{2} 24 ; z_{3} 12 ; z_{4} 23$ \\
\hline Dimensionless phase $\varphi^{[2]}$ & 0.5 & 0.5 \\
\hline Periodicity $T^{(2)}$ & $1 / 12 n_{2}$ & $1 / 12 n_{2}$ \\
\hline Contact ratio $\varepsilon$ & $\varepsilon_{1} 1.5111, \varepsilon_{2} 1.5111$ & $\varepsilon_{1} 1.4990, \varepsilon_{2} 1.5060$ \\
\hline
\end{tabular}

The Varying regularity of optimal phase angle of gear ratio 3 is similar to that of gear ratio 1 according to Fig. 10. According to the relevant parameters in Table 3, $\varphi^{[2]}$, and $T^{(2)}$ are all equal, and varying regularity of optimal phase angle is roughly similar, where the subtle differences are due to the different contact ratio $\varepsilon$ causing the different percentage of $k$ and $2 k$. 


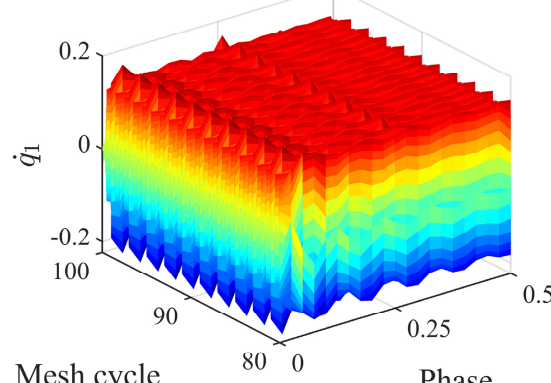

(a)

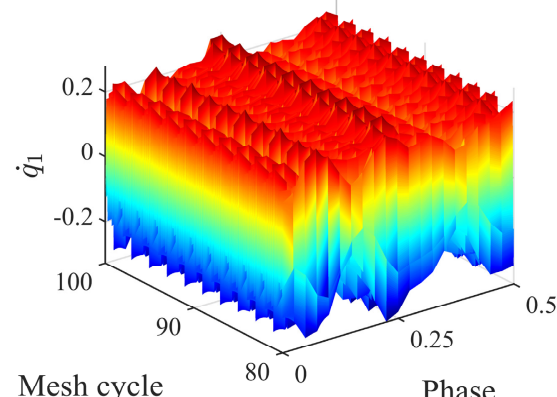

(d)

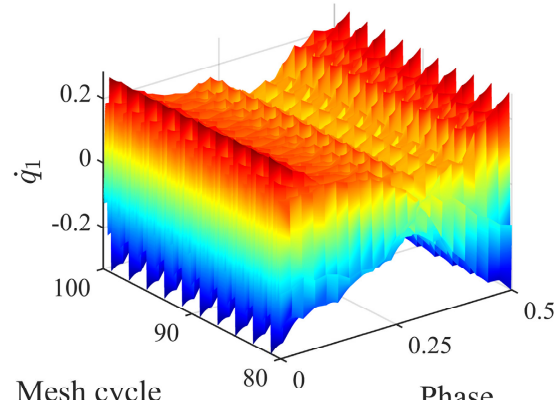

(g)

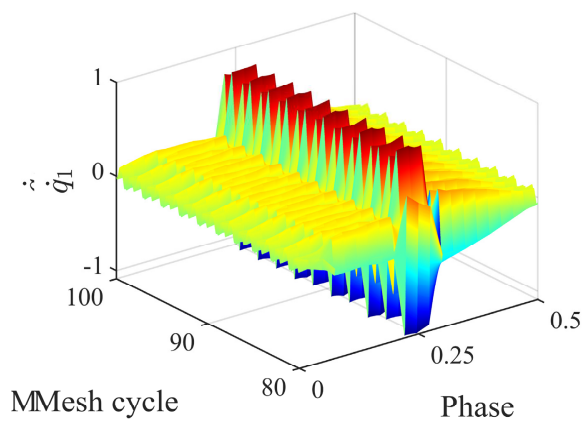

(j)

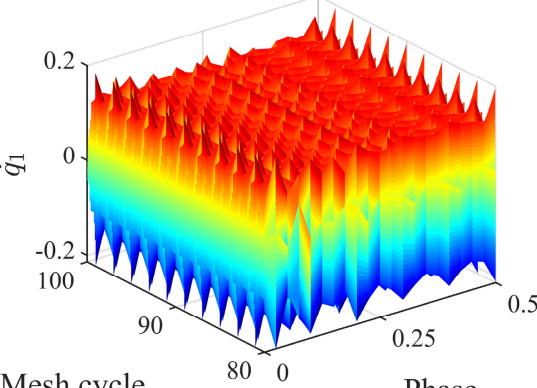

(b)

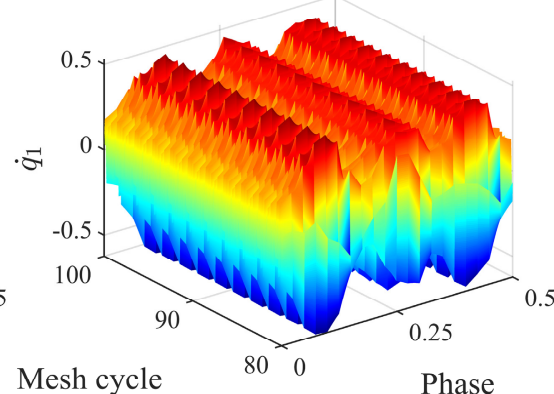

(e)

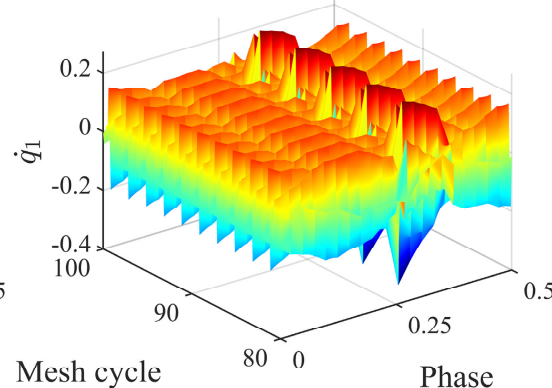

(h)

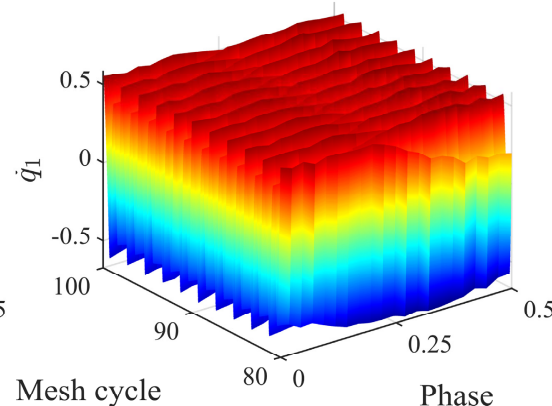

(k)

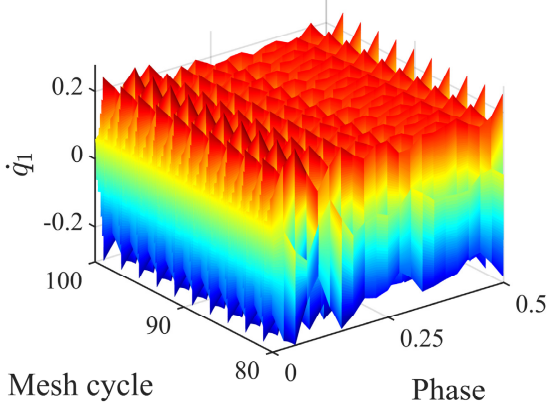

(c)

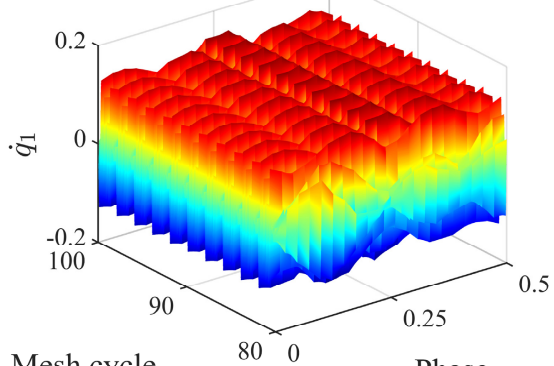

(f)

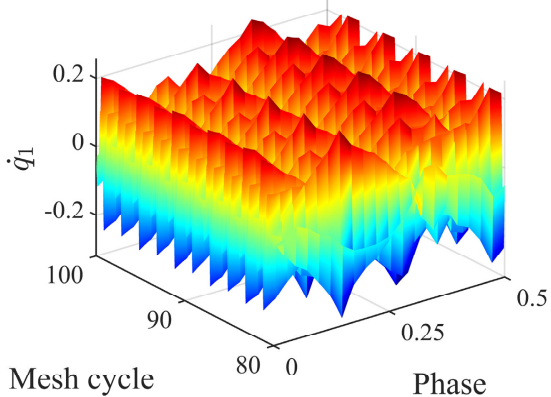

(i)

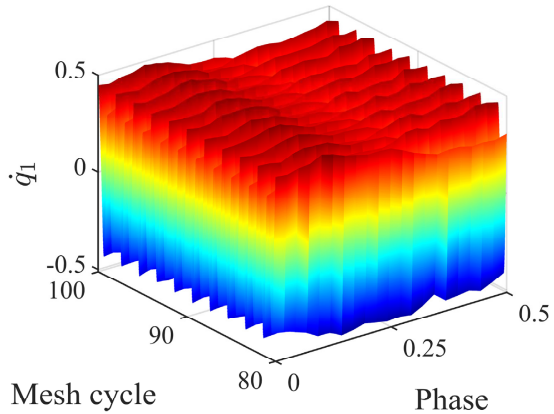

(1)

Fig. 11 Effect of phase configuration on vibration curve for gear ratio 3 (a) $\Omega_{1}=0.05$ (b)
$\Omega_{1}=0.1$ (c) $\Omega_{1}=0.15$
(d) $\Omega_{1}=0.25$
(e) $\Omega_{1}=0.30$
(f) $\Omega_{1}=0.45$
(g) $\Omega_{1}=0.5$

$$
\Omega_{1}=0.6
$$
(j) $\Omega_{1}=0.8$
(k) $\Omega_{1}=1.0$
(l) $\Omega_{1}=1.15$

For a detailed comparison, the vibration curve of different phase angles between $\Omega_{1}=0.05$ and $\Omega_{1}=1.4$ is calculated with the mesh frequency of $\Omega_{1}=0.05$ as the interval. Some of these results are shown in Fig. 11. It is obvious that there are several optimal phase angles in Fig. 11(a), (b), (c), (d). At $\Omega_{1}=0.3$ ( the $1 / 3$ super-harmonics resonance of the II stage), there are clearly 3 optimal phase 
angles. At $\Omega_{1}=0.5$ ( the $1 / 2$ super-harmonics resonance of the II stage), phase has a significant dampening effect on resonance. At $\Omega_{1}=0.8$, the system is in the non-linear jump region and the change in phase gives the gears no impact and single-sided impact motion. When $\Omega_{1}>1$, the effect of phase on the system diminishes significantly.

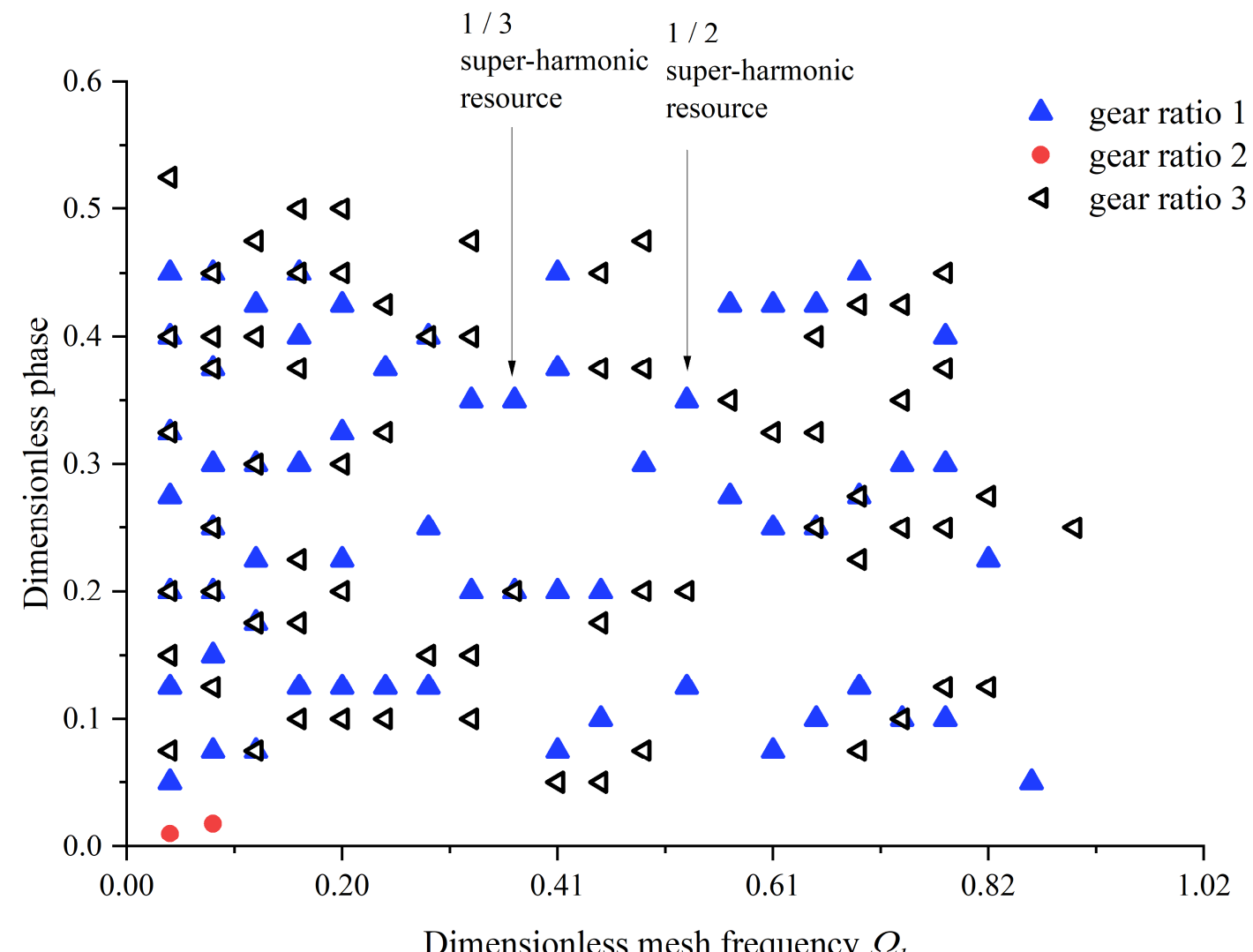

Fig. 12 Distribution of optimal phase angle

The distribution of the optimal phase angle is shown in Fig. 12, based on local minimum of RMS of $\dot{q}_{1}$ at the three gear ratios from $\Omega_{1}=0.05$ to $\Omega_{1}=1.4$. The varying regularity of steady state is in general agreement with the previous analysis and Figure 13 gives a more accurate value for the optimal phase angle. The number of optimal phase angles of the gear ratio 2 is always 1, which the phase fluctuates with the dimensionless mesh frequency. Next, we focus on the optimal phase angle distribution of gear ratio 1 and gear 3. Below the mesh frequency at the $1 / 3$ super-harmonics resonance of the I stage, the number of optimal phase angles decreases as the mesh frequency increases. Beyond $\Omega_{1}=0.33$, the number of the optimal phase angles increases instantly and then decreases as the mesh frequency increases at $\Omega_{1} \in[0.33,0.5]$. At $\Omega_{1} \in[0.5,0.8]$, the optimal phase angle becomes multiple and fluctuates. $\Omega_{1} \in[0.5,0.8]$. When $\Omega_{1}>0.85$, the phase has little effect on the vibration characteristics and the optimal phase angle cannot be obtained when calculation errors are taken into account. There $\Omega_{1}=0.85$ is defined as the critical value of gear ratio 1 and gear ratio 3 . 


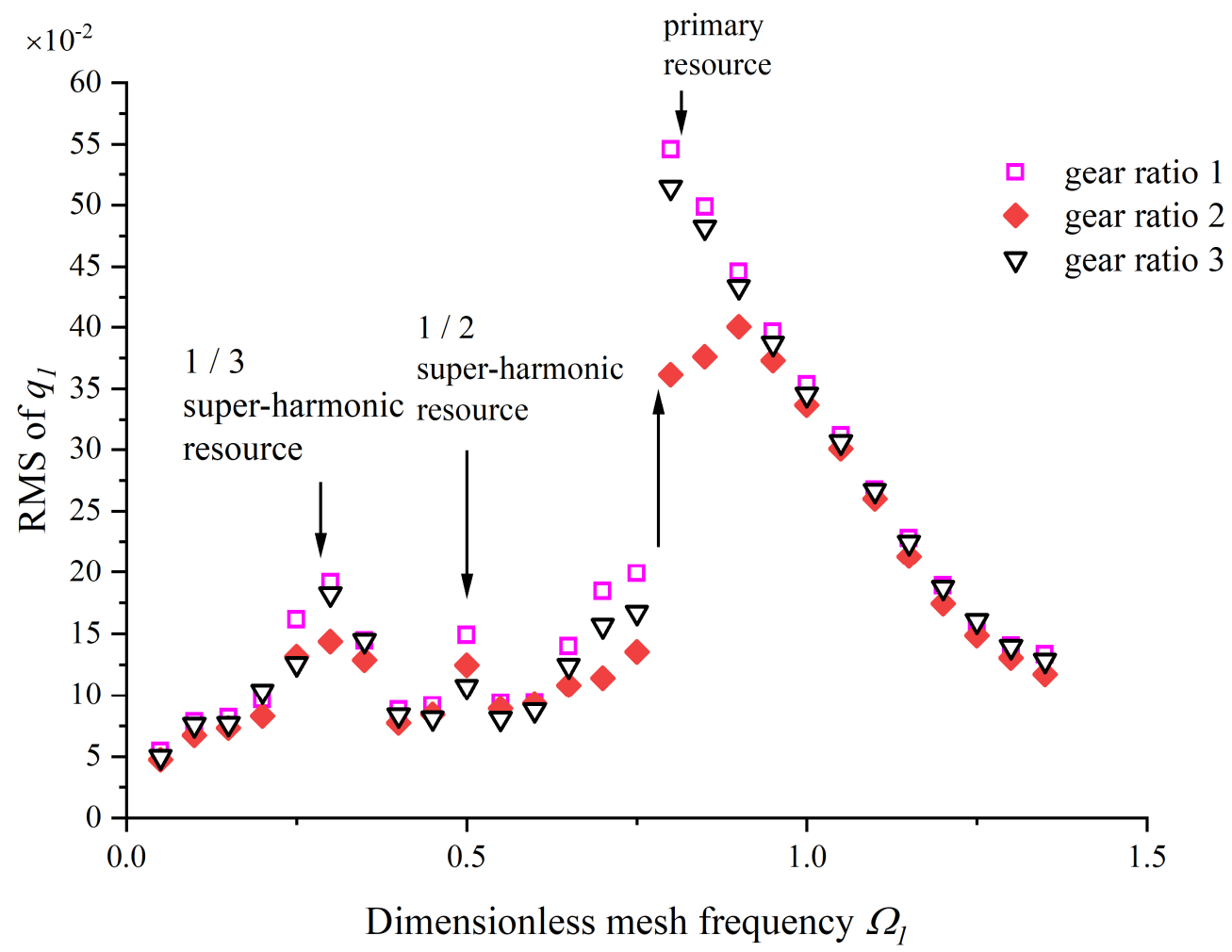

Fig. 13 Comparison of maximum RMS of $\dot{q}_{1}$

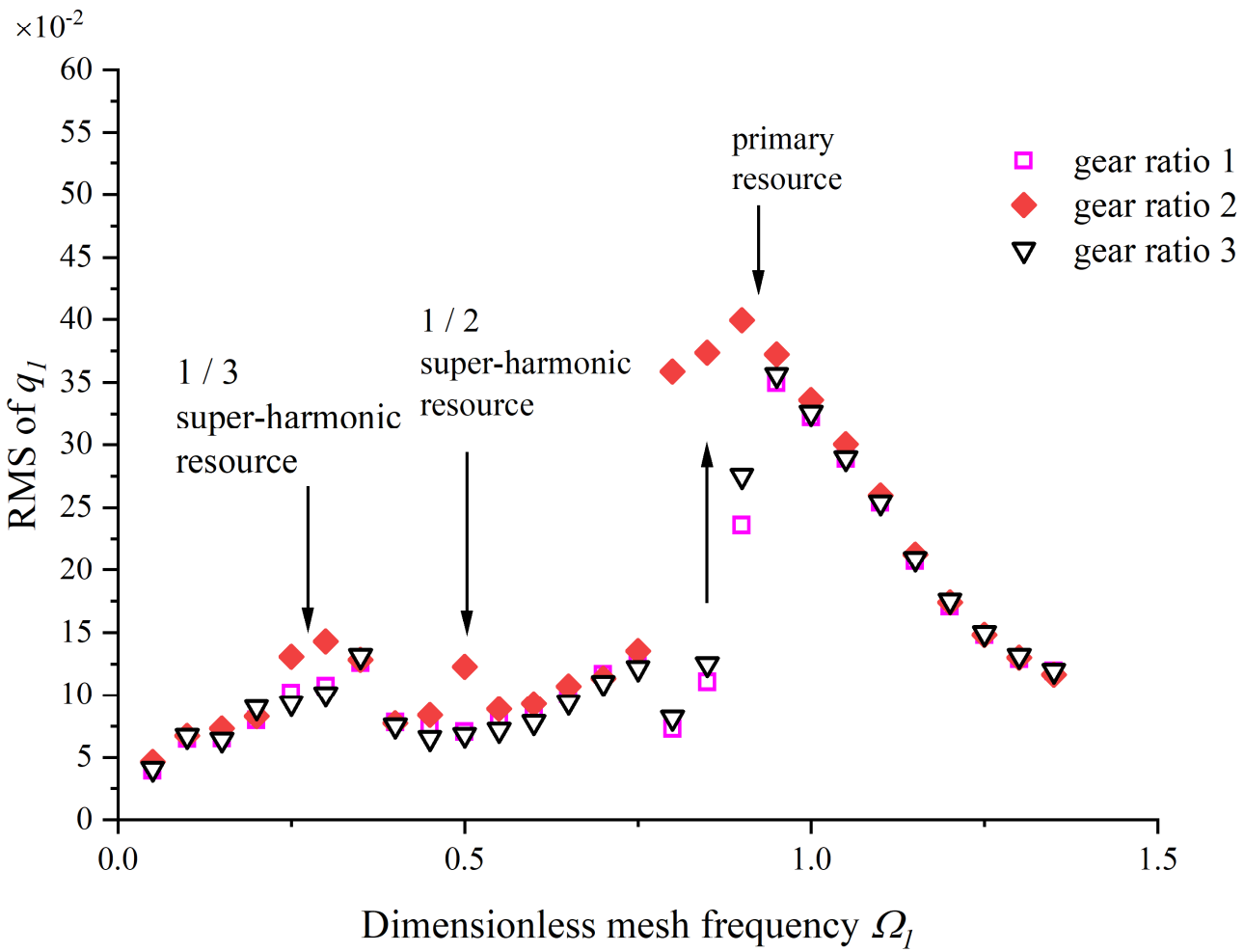

Fig. 14 Comparison of minimum RMS of $\dot{q}_{1}$ 
In order to obtain the comparative influence of the phase on the magnitude of vibration, the RMS of the different phase angles of the three gear ratios are the maximum value (Fig. 13) and the minimum value of optimal phase angles (Fig. 14). The primary resonance point, the $1 / 2$ super-harmonic resonance point, and the 1/3 super-harmonic resonance point can be clearly obtained from the Fig. 13 and Fig. 14. The maximum value of gear ratio 2 is significantly lower than gear ratio 1 and gear 3 in the steady state. By comparing the minimum values of RMS of $\dot{q}_{1}$, it can be seen that the minimum amplitudes of the optimal phase angle of gear ratio 1 and gear ratio 3 are better than those of gear ratio 2. Comparing Figure 13 and Fig. 14, the difference between the mean and minimum values for gear ratio 2 is significantly smaller than for gear ratio 1 and gear ratio 3 . The optimal phase angle is better optimized for gear ratio 1 and gear ratio 3 than for gear ratio 2. Without optimal phase configuration, gear ratio 2 can be used in preference.

\section{Conclusion}

Through the meshing theory analysis and dynamic analysis of the multi-stage gear transmission, the phase configuration theory is proposed. The complete meshing space description of the multi-stage gear transmission system is derived. The dynamic model based on phase configuration theory is established. The influence regularity of gear ratio and phase on the system is revealed according to its vibration characteristics.

(1) Based on the two-stage gear transmission system, the phase configuration theory is established, and the complete description of meshing space is obtained. The phase configuration theory is extended to the n-stage gear transmission system, and the complete description of n-stage meshing space based on phase angle and time is derived, which provides a theoretical basis for analyzing the phase configuration dynamics of the multi-stage gear transmission system.

(2) The influence of phase configuration on the vibration characteristics of a two-stage gear transmission system is explored. After numerical calculations, there is an optimal phase angle to minimize the vibration intensity. The position of the optimal phase can be determined according to the FFT spectrum and RMS of vibration.

(3) When the numbers of intermediate shaft teeth are not the coprime number, the range of phase angle is large. There are multiple optimal phase angles at low mesh frequency. With the increase of mesh frequency, the number of optimum phase angles decreases. When the mesh frequency exceeds the critical value, the optimal phase angle is nonexistent. At this time, the phase angle has little effect on the vibration characteristics.

(4) When the numbers of intermediate shaft teeth are the coprime number, the range of phase angle is small. There is only one optimal phase angle position at low mesh frequency. In the absence of optimal phase configuration, the vibration intensity of the coprime number of teeth of the intermediate shaft is less than that of the non-coprime.

\section{Data Availability Statements}

The datasets generated during and/or analyzed during the current study are available from the corresponding author on reasonable request.

\section{Conflict of interests}

Wei Li and other co-authors declare that we have no known competing financial interests or personal relationships that could have appeared to influence the work reported in this paper.

\section{Acknowledgements}

The authors would like to acknowledge the financial support from the NSFC, the research is funded by National Natural Science Foundation of China (contract No. 51775036), these supports are gracefully acknowledged. 


\section{References}

1. Wang, Y., Zhang, W.J.: Stochastic Vibration Model of Gear Transmission Systems Considering Speed-Dependent Random Errors. Nonlinear Dyn. 17, 187-203 (1998). https://doi.org/10.1023/A:1008389419585

2. Roda-Casanova, V., Gonzalez-Perez, I.: Investigation of the effect of contact pattern design on the mechanical and thermal behaviors of plastic-steel helical gear drives. Mech. Mach. Theory. 164, 104401 (2021). https://doi.org/10.1016/j.mechmachtheory.2021.104401

3. Bobach, L., Beilicke, R., Bartel, D.: Transient thermal elastohydrodynamic simulation of a spiral bevel gear pair with an octoidal tooth profile under mixed friction conditions. Tribol. Int. 143, 106020 (2020). https://doi.org/10.1016/j.triboint.2019.106020

4. Hu, B., Zhou, C., Wang, H., Chen, S.: Nonlinear tribo-dynamic model and experimental verification of a spur gear drive under loss-of-lubrication condition. Mech. Syst. Signal Process. 153, (2021). https://doi.org/10.1016/j.ymssp.2020.107509

5. Liu, H., Yan, P., Gao, P.: Effects of temperature on the time-varying mesh stiffness, vibration response, and support force of a multi-stage planetary gear. J. Vib. Acoust. Trans. ASME. 142, 1-15 (2020). https://doi.org/10.1115/1.4047246

6. Raclot, J.P., Velex, P.: Simulation of the dynamic behaviour of single and multi-stage geared systems with shape deviations and mounting errors by using a spectral method. J. Sound Vib. 220, 861-903 (1999). https://doi.org/10.1006/jsvi.1998.1988

7. Brecher, C., Schroers, M., Löpenhaus, C.: Experimental analysis of the dynamic noise behavior of a two-stage cylindrical gearbox. Prod. Eng. 11, 695-702 (2017). https://doi.org/10.1007/s11740-017-0775-y

8. Wang, X.: Stability research of multistage gear transmission system with crack fault. J. Sound Vib. 434, 63-77 (2018). https://doi.org/10.1016/j.jsv.2018.07.037

9. Yi, Y., Qin, D., Liu, C.: Investigation of electromechanical coupling vibration characteristics of an electric drive multistage gear system. Mech. Mach. Theory. 121, 1339-1351 (2018). https://doi.org/10.1016/j.mechmachtheory.2017.11.011

10. Yavuz, S.D., Saribay, Z.B., Cigeroglu, E.: Nonlinear dynamic analysis of a drivetrain composed of spur, helical and spiral bevel gears. Nonlinear Dyn. 100, 3145-3170 (2020). https://doi.org/10.1007/s11071-020-05666-8

11. Lu, W., Zhang, Y., Cheng, H., Zhou, Y., Lv, H.: Research on dynamic behavior of multistage gears-bearings and box coupling system. Meas. J. Int. Meas. Confed. 150, 107096 (2020). https://doi.org/10.1016/j.measurement.2019.107096

12. Zhang, M., Zhang, Z., Shi, L., Gao, P., Zhang, J., Zhang, W.: A new assembly error modeling and calculating method of complex multi-stage gear transmission system for a large space manipulator. Mech. Mach. Theory. 153, 103982 (2020). https://doi.org/10.1016/j.mechmachtheory.2020.103982

13. Cao, Z., Chen, Z., Jiang, H.: Nonlinear dynamics of a spur gear pair with force-dependent mesh stiffness. Nonlinear Dyn. 99, 1227-1241 (2020). https://doi.org/10.1007/s11071-01905348-0

14. Litak, G., Friswell, M.I.: Dynamics of a gear system with faults in meshing stiffness. Nonlinear Dyn. 41, 415-421 (2005). https://doi.org/10.1007/s11071-005-1398-y

15. Xiao, W., Li, J., Pan, T., Zhang, X., Huang, Y.: Investigation into the influence of particles' friction coefficient on vibration suppression in gear transmission. Mech. Mach. Theory. 108, 217-230 (2017). https://doi.org/10.1016/j.mechmachtheory.2016.10.015

16. Xiao, W., Chen, Z., Pan, T., Li, J.: Research on the impact of surface properties of particle on damping effect in gear transmission under high speed and heavy load. Mech. Syst. Signal Process. 98, 1116-1131 (2018). https://doi.org/10.1016/j.ymssp.2017.05.021

17. Guo, Y., Parker, R.G.: Analytical determination of mesh phase relations in general compound 
planetary gears. Mech. Mach. Theory. 46, 1869-1887 (2011).

https://doi.org/10.1016/j.mechmachtheory.2011.07.010

18. Wang, S., Huo, M., Zhang, C., Liu, J., Song, Y., Cao, S., Yang, Y.: Effect of mesh phase on wave vibration of spur planetary ring gear. Eur. J. Mech. A/Solids. 30, 820-827 (2011). https://doi.org/10.1016/j.euromechsol.2011.06.004

19. Peng, D., Smith, W.A., Randall, R.B., Peng, Z.: Use of mesh phasing to locate faulty planet gears. Mech. Syst. Signal Process. 116, 12-24 (2019).

https://doi.org/10.1016/j.ymssp.2018.06.035

20. Peng, D., Smith, W.A., Borghesani, P., Randall, R.B., Peng, Z.: Comprehensive planet gear diagnostics: Use of transmission error and mesh phasing to distinguish localised fault types and identify faulty gears. Mech. Syst. Signal Process. 127, 531-550 (2019). https://doi.org/10.1016/j.ymssp.2019.03.024

21. Wang, C., Parker, R.G.: Dynamic modeling and mesh phasing-based spectral analysis of quasi-static deformations of spinning planetary gears with a deformable ring. Mech. Syst. Signal Process. 136, 106497 (2020). https://doi.org/10.1016/j.ymssp.2019.106497

22. Sanchez-Espiga, J., Fernandez-del-Rincon, A., Iglesias, M., Viadero, F.: Planetary gear transmissions load sharing measurement from tooth root strains: Numerical evaluation of mesh phasing influence. Mech. Mach. Theory. 163, 104370 (2021). https://doi.org/10.1016/j.mechmachtheory.2021.104370

23. Kahraman, A., Singh, R.: NON-LINEAR DYNAMICS OF A SPUR GEAR PAIR. J. Sound Vib. 49-75 (1990)

24. Kahraman, A., Blankenship, G.W.: Effect of involute tip relief on dynamic response of spur gear pairs. J. Mech. Des. Trans. ASME. 121, 313-315 (1999). https://doi.org/10.1115/1.2829460

25. Celikay, A., Donmez, A., Kahraman, A.: An experimental and theoretical study of subharmonic resonances of a spur gear pair. J. Sound Vib. 515, (2021). https://doi.org/10.1016/j.jsv.2021.116421

26. Li, W., Sun, J., Yu, J.: Analysis of dynamic characteristics of a multi-stage gear transmission system. JVC/Journal Vib. Control. 25, 1653-1662 (2019).

https://doi.org/10.1177/1077546319830810 